\title{
Aging of target lipid parameters in fingermark residue using GC/MS: Effects of influence factors and perspectives for dating purposes
}

\author{
Aline Girod *, Alexandra Spyratou, David Holmes, Céline Weyermann \\ Ecole des Sciences Criminelles, University of Lausanne, Batochime, CH 1015 Lausanne, Switzerland
}

\section{A R T I C L E I N F O}

\section{Article history:}

Received 24 May 2015

Received in revised form 14 December 2015

Accepted 16 December 2015

Available online $\mathrm{xxxx}$

\section{Keywords:}

Fingerprint

Aging model

Classification

Regression

Bayesian network

Blind tests

\begin{abstract}
A B S T R A C T
Despite the recurrence of fingermark dating issues and the research conducted on fingermark composition and aging, no dating methodology has yet been developed and validated. In order to further evaluate the possibility of developing dating methodologies based on the fingermark composition, this research proposed an in-depth study of the aging of target lipid parameters found in fingermark residue and exposed to different influence factors. The selected analytical technique was gas chromatography coupled with mass spectrometry (GC/MS). The effects of donor, substrate and enhancement techniques on the selected parameters were firstly evaluated. These factors were called known factors, as their value could be obtained in real caseworks. Using principal component analysis (PCA) and univariate exponential regression, this study highlighted the fact that the effects of these factors were larger than the aging effects, thus preventing the observation of relevant aging patterns. From a fingermark dating perspective, the specific value of these known factors should thus be included in aging models newly built for each case. Then, the effects of deposition moment, pressure, temperature and lighting were also evaluated. These factors were called unknown factors, as their specific value would never be precisely obtained in caseworks. Aging models should thus be particularly robust to their effects and for this reason, different chemometric tools were tested: PCA, univariate exponential regression and partial least square regression (PLSR). While the first two models allowed observing interesting aging patterns regardless of the value of the applied influence factors, PLSR gave poorer results, as large deviations were obtained. Finally, in order to evaluate the potential of such modelling in realistic situations, blind analyses were carried out on eight test fingermarks. The age of five of them was correctly estimated using soft independent modelling of class analogy analysis (SIMCA) based on PCA classes, univariate exponential linear regression and PLSR. Furthermore, a probabilistic approach using the calculation of likelihood ratios (LR) through the construction of a Bayesian network was also tested. While the age of all test fingermarks were correctly evaluated when the storage conditions were known, the results were not significant when these conditions were unknown. Thus, this model clearly highlighted the impact of storage conditions on correct age evaluation.

This research showed that reproducible aging modelling could be obtained based on fingermark residue exposed to influence factors, as well as promising age estimations. However, the proposed models are still not applicable in practice. Further studies should be conducted concerning the impact of influence factors (in particular, storage conditions) in order to precisely evaluate in which conditions significant evaluations could be obtained. Furthermore, these models should be properly validated before any application in real caseworks could be envisaged.
\end{abstract} (c) 2015 The Chartered Society of Forensic Sciences. Published by Elsevier Ireland Ltd. All rights reserved.

\section{Introduction}

Fingermarks have been commonly used in forensic investigations for more than a century, essentially for identification purposes [1-4]. However, other useful information could be extracted from fingermarks. For example, the estimation of their age, i.e. the time of their transfer, can be crucial for the resolution of cases. Indeed, this information is directly linked to the relevance of these marks, as it allows determination of whether they were left during the crime or not $[5,6]$. It is not uncommon

\footnotetext{
* Corresponding author
}

that an identified suspect admits to having been at a crime scene, but claims that the contact occurred at a time different to that of the crime and for legitimate reasons. Despite the recurrence of such issues and the research conducted on this topic, no fingermark dating methodology has yet been validated by the entire forensic community [7-9].

Among the fingermark dating methodologies proposed so far, the study of the aging of chemical characteristics seems to offer good perspectives because numerous target compounds can be studied using various analytical techniques, as already foreseen by Olsen [10]. Later, this opinion was also supported by Wertheim, who saw in the analysis of fingermark constituents the opportunity to find compounds deteriorating over time without being too much affected by influence factors,

http://dx.doi.org/10.1016/j.scijus.2015.12.004

1355-0306/@ 2015 The Chartered Society of Forensic Sciences. Published by Elsevier Ireland Ltd. All rights reserved.

Please cite this article as: A. Girod, et al., Aging of target lipid parameters in fingermark residue using GC/MS: Effects of influence factors and perspectives for dating purpo..., Sci. Justice (2016), http://dx.doi.org/10.1016/j.scijus.2015.12.004 
which would allow for accurate age estimations [11]. This idea was also mentioned in different reviews on the subject [12-14]. Studies reported that lipids were interesting targets for aging research, as they can be analysed by techniques commonly available in forensic laboratories (e.g., GC/MS or FTIR) and undergo oxidation over time [7-9,12,15-17]. Furthermore, reproducible aging patterns following an exponential decay could be detected, particularly for fatty acids, cholesterol and squalene extracted from fingermarks stored under controlled conditions and analysed using GC/MS [16,17]. However, it was also highlighted that the initial composition and aging of lipids was significantly affected by different influence factors, classified in five groups: the donor, the substrate, the enhancement techniques, the deposition and the storage conditions [12]. Some research using GC/MS were already conducted to study the impact of the donor [17-20], the substrate [17], the application of enhancement technique [19] and the exposition to light [16] on the initial composition and aging of some lipids (particularly fatty acids, squalene and cholesterol). Overall, large variability was observed. The calculation of ratios of compounds using squalene, cholesterol and wax esters was proposed to reduce part of the variability due to donors, substrate and enhancement techniques [17,19]. However, the efficiency of these ratios still needs to be further tested on larger sets of fingermarks exposed to various influence factors. Based on recent papers proposing a formal practical fingermark dating framework [8,9], the five groups of influence factors mentioned above can be further categorised as known or unknown factors. In fact, as it seems reasonable to consider that fingermark dating questions would mostly be raised after the collection, enhancement and identification of the questioned marks, donor, substrate and enhancement techniques can be considered as known influence factors. Thus, specific aging models could be built for each new case taking these particular parameters into account, i.e. using comparison fingermarks collected from the identified donor, on the same substrate and applying the same enhancement techniques to the comparison and questioned marks. The variability brought by these three sources could thus be limited. On the contrary, deposition and storage conditions can generally be considered as unknown factors, as they will never be precisely obtained in caseworks. For fingermark dating perspective, it is therefore particularly important to study the effects of these factors on target compounds (e.g., lipids) and take them into account in aging models.

The aim of the present research was to study the aging of target lipid parameters available in fingermark residue and exposed to different influence factors using GC/MS. Whether the residue variability reported in the literature could be reduced in such a way that robust aging models could be developed and used for fingermark dating perspectives was assessed. First, the effects of known factors on selected lipid parameters were evaluated over time using principal component analysis (PCA) and univariate regression, in order to collect fundamental knowledge about the variability brought by such factors. Four donors, two substrates (microfiber filters and glass) and one enhancement technique (aluminium fingermark powder, also called Argentorat) were tested. Then, the effects of unknown factors on lipid parameters were also studied over time with PCA and univariate regression. Partial least square regression (PLSR) was also used in order to evaluate if multivariate regression was more appropriate to model aging affected by these factors. Two different deposition moments (three months apart) and three different deposition pressures ( $100 \mathrm{~g}, 500 \mathrm{~g}, 1000 \mathrm{~g})$ were tested, as well as three different storage temperatures $\left(15,20\right.$ and $\left.25^{\circ} \mathrm{C}\right)$ and two different lighting conditions (darkness and light). Furthermore, in order to evaluate the real potential of such modelling for fingermark dating purposes, blind analyses of eight test fingermarks were carried out and their age was estimated using the relevant PCA, univariate regression and PLSR models. Finally, based on literature on the dating of ink traces [21,22], gunshot residue [23] and fingermarks [8,9], a probabilistic approach using the calculation of likelihood ratios (LR) obtained from a Bayesian network was also tested to evaluate the age of these eight test fingermarks.

\section{Material and methods}

\subsection{Sampling: donors, deposition protocol and data sets}

Four different donors were selected for this study, based on their availability during the different research phases. All donors were Caucasian, non-smoker and omnivores who had no diagnosed metabolic disease. Furthermore, these donors were classified based on a recent study proposing a donor classification based on their fingermark lipid composition [20] (Table 1).

All the fingermarks collected for this study were deposited on $25 \mathrm{~mm}$ diameter glass microfiber filters (Whatman, Bottmingen, Switzerland) or on glass microscope slides (VWR international SA, China) according to the following deposition protocol:

1. The donors were asked to follow their tasks normally before deposition. The only condition was to avoid hand washing with soap within the last 45 min preceding the deposition.

2. Both thumbs were gently rubbed on the forehead, miming a natural movement.

3. The time of deposition was maintained at $15 \mathrm{~s}$ and each fingermark was deposited on a kitchen scale to obtain the desired pressure (variations of $\pm 50 \mathrm{~g}$ were observed). During each deposition session, the right and left thumbs were collected.

After deposition, some fingermarks were directly analysed $(t=5-$ $10 \mathrm{~min}$ ) and others were aged under different storage conditions up to around one month before analysis. For each age, two fingermark specimens were analysed. As mentioned in the introduction, seven influence factors were studied in this work (i.e. donor, substrate, enhancement, deposition moment, deposition pressure, temperature and lighting) and blind tests were also conducted. Table 2 summarised the acquired data sets.

When one influence factor was tested, the others were fixed to the following standard values (unless other precisions are given under Description in Table 2):

- Substrate: paper

- Enhancement: none

- Deposition moment: fingermarks used in a specific data set were left within one month

- Pressure: $500 \mathrm{~g}$

- Temperature: $20^{\circ} \mathrm{C}$ (climate chamber)

- Lighting: complete darkness.

It is important to note that the effects of enhancement techniques (set \#3) were tested using the Argentorat powder (Paine Spurensicherung, Arni, $\mathrm{CH}$ ) because this technique is one of the most widely used on non-porous surfaces such as glass [4]. The application of the powder was made when the fingermarks reached the desired age and analysis was then conducted around 30 min after enhancement. Concerning the lighting conditions (sets \#3 and 7), the fingermarks stored in the dark were put in closed boxes kept in an air-conditioned laboratory. The fingermarks exposed to light were stored in an open box in the same laboratory close to the window, being thus exposed to light during the day and to darkness during the night (normal $24 \mathrm{~h}$ cycle). Finally, it should be highlighted that, as the analyses were

Table 1

Characteristics of the fingermark donors.

\begin{tabular}{lllll}
\hline Name & Donor classification & Sex & Age & Cosmetics \\
\hline A & Medium-rich & F & 26 & No \\
B & Medium-rich & M & 27 & Face cream \\
C & Medium-poor & F & 27 & Face cream \\
D & Poor & F & 32 & No \\
\hline
\end{tabular}


Table 2

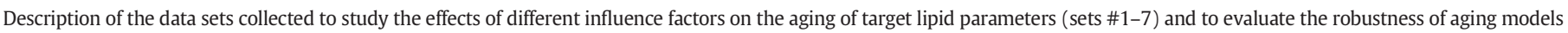
using test fingermarks of unknown ages (set \#8).

\begin{tabular}{|c|c|c|c|}
\hline \# sets & Tested factors & Description & Aging [days] \\
\hline \multirow[t]{3}{*}{1} & Donors & Donors A and B: medium-rich & $0-33$ \\
\hline & 3 different donor types & Donor C: medium-poor & $0-28$ \\
\hline & & Donor D: poor & $0-27$ \\
\hline \multirow[t]{2}{*}{2} & Substrates & Donors A and B & $0-33$ \\
\hline & Microfiber filters or glass & & \\
\hline \multirow[t]{2}{*}{3} & Enhancement & Donor C & $0-36$ \\
\hline & Powdered with Argentorat or not enhanced & Fingermarks left on glass and stored in the dark or exposed to a normal $24 \mathrm{~h}$ cycle & \\
\hline \multirow[t]{2}{*}{4} & Deposition moments & Donor C & $0-24$ \\
\hline & In April 2012 or in July 2012 & & \\
\hline \multirow[t]{2}{*}{5} & Pressure & Donor D & $0-29(1000 \mathrm{~g})$ \\
\hline & $100 \mathrm{~g}, 500 \mathrm{~g}$ or $1000 \mathrm{~g}$ & & $0-27(100+500 g)$ \\
\hline \multirow[t]{2}{*}{6} & Temperature & Donors A and B & $0-35\left(20+25^{\circ} \mathrm{C}\right)$ \\
\hline & $15^{\circ} \mathrm{C}, 20^{\circ} \mathrm{C}$ or $25^{\circ} \mathrm{C}$ & Fingermarks left on paper or glass within 3 months & $0-32\left(15^{\circ} \mathrm{C}\right)$ \\
\hline \multirow[t]{2}{*}{7} & Lighting & Donor C & $0-36$ \\
\hline & Dark or normal 24 h cycle & $\begin{array}{l}\text { Fingermarks left on glass and not enhanced or powder-enhanced } \\
\text { (This set actually corresponds to set \#3) }\end{array}$ & \\
\hline \multirow[t]{4}{*}{8} & Blind tests & Donor C & Unknown \\
\hline & 8 test fingermarks & $\begin{array}{l}\text { Fingermarks left on glass and stored in an office under unknown temperature and lighting } \\
\text { conditions. }\end{array}$ & Max. 36 days \\
\hline & & Ages evaluated using models of set \#7 without enhancement, as this set corresponds to the & \\
\hline & & known influence factors of the test fingermarks (i.e. donor, substrate and enhancement) & \\
\hline
\end{tabular}

conducted using GC/MS, the fingermark specimens were destroyed. New specimens were thus analysed for each age group. This added some uncontrollable variability to each data set, due to the variations of the initial composition of fingermarks $[12,15,20]$.

\subsection{Fingermark analysis and target compounds (procedures based on [20])}

For extraction of the fingermarks left on microfiber filters, the substrates were immersed in capped glass vials $(32 \mathrm{~mm} \times 11.6 \mathrm{~mm}$, purchased from Laubscher, Geneva, Switzerland) filled with $1.5 \mathrm{~mL}$ dichloromethane (99.99\%, purchased from Fisher Scientific, Wohlen, Switzerland) for $60 \mathrm{~s}$ and then removed. To extract the fingermarks left on glass, a microfiber filter was taken using forceps, immersed in dichloromethane and rubbed against the glass surface in order to collect the fingermark residue. The microfiber filter was then immersed in a capped glass vial filled with $1.5 \mathrm{~mL}$ dichloromethane for $60 \mathrm{~s}$ and removed. A second microfiber filter was taken with forceps and dry rubbed against the glass to maximise the amount of the extracted residue. This second microfiber filter was also immersed in the same vial as previously described, for $60 \mathrm{~s}$ and removed. In order to concentrate the extracted residue, the dichloromethane was evaporated from each sample under a stream of nitrogen until only a few microliters were left. The remaining liquid was transferred into an insert of $50 \mathrm{~mL}$ installed on a spring (inserts and springs purchased from Laubscher, Geneva, Switzerland) and evaporated to dryness under a stream of nitrogen. The residue was then dissolved in $20 \mathrm{~mL}$ of dichloromethane containing an internal standard $(0.05 \mathrm{mg} / \mathrm{mL}$ of 1-decanol, purchased from SigmaAldrich, Buchs, SG, Switzerland). The internal standard was chosen based on its retention time and its similarity with the targeted compounds.

For powder-enhanced fingermarks on glass, the same steps were conducted until the second microfiber filter was removed; a filtration step was included at this point in order to avoid injecting carbon contaminants on the GC column. The dichloromethane containing the extracted residue was thus first put into a syringe with a head filter of RC $0.45 \mu \mathrm{m}$ provided by Laubscher (Geneva, Switzerland) and then injected into a new, clean vial before concentration and inclusion of the internal standard.

Blank samples (clean microfiber filters and clean microscope slides) were also extracted and analysed for each analysis run.

The composition of the collected fingermarks was analysed using a gas chromatograph Clarus 500 equipped with an autosampler and coupled with a mass spectrometer Clarus 560 in electron impact (EI) mode maintained at $230^{\circ} \mathrm{C}$, both instruments from PerkinElmer. The column used for the analysis was an HP5-MS, $30 \mathrm{~m} \times 0.25 \mathrm{~mm} \times$ $0.25 \mathrm{~mm}$ from Agilent and the carrier gas was helium with a constant flow of $1 \mathrm{~mL} / \mathrm{min}$. Sample quantity of $2 \mathrm{~mL}$ was injected in splitless mode using a purge time of $1.5 \mathrm{~min}$ through the injector maintained at a temperature of $250{ }^{\circ} \mathrm{C}$. The temperature program used was: $80^{\circ} \mathrm{C}$ for $1 \mathrm{~min}$, followed by an increase from 80 to $230{ }^{\circ} \mathrm{C}$ at a rate of $10{ }^{\circ} \mathrm{C} / \mathrm{min}$, isothermal step at $230^{\circ} \mathrm{C}$ held for $2 \mathrm{~min}$, an increase from 230 to $290{ }^{\circ} \mathrm{C}$ at a rate of $6{ }^{\circ} \mathrm{C} / \mathrm{min}$ and then from 290 to $320{ }^{\circ} \mathrm{C}$ at a rate of $3{ }^{\circ} \mathrm{C} / \mathrm{min}$ and finally a last isothermal step at $320^{\circ} \mathrm{C}$ for $2 \mathrm{~min}$. A solvent delay of $3.6 \mathrm{~min}$ was applied and the transfer line temperature was maintained at $300{ }^{\circ} \mathrm{C}$. The mass analyser used was a quadrupole maintained at $150{ }^{\circ} \mathrm{C}$ and set in scan mode between 40 and $550 \mathrm{~m} / \mathrm{z}$.

To ensure the result quality, a control solution containing representative lipids and the internal standard (1-decanol, $0.05 \mathrm{mg} / \mathrm{mL}$ ) was injected and analysed every week. Control charts were maintained in accordance with the literature $[24,25]$. The selected representative compounds were squalene $(1 \mathrm{mg} / \mathrm{mL})$, cholesterol $(1 \mathrm{mg} / \mathrm{mL})$, myristyl palmitoleate $(0.1 \mathrm{mg} / \mathrm{mL})$, and palmityl palmitate $(0.1 \mathrm{mg} / \mathrm{mL})$. The sterols were purchased from Sigma-Aldrich (Buchs, SG, Switzerland) and the wax esters from Nu-Chek Prep (Elysian, MN).

Compounds found in the chromatograms were qualitatively identified using mass spectra information, comparison with reference substances and the NIST05 database. Ten target lipids proposed in a previous research [20] were studied in this work, as they were identified in all analysed fingermarks, showed reasonable abundance $(\mathrm{S} / \mathrm{N}>10)$ and good resolution. These characteristics indicate good target compounds for fingermark dating purposes [8,9]. Furthermore, three squalene derivatives (SD1, SD2 and SD3) that were also identified in this previous study were added for a total of 13 target lipids, called aging variables or parameters hereafter (Table 3 ). Target ions were selected for each compound to be able to identify them automatically in all chromatograms. The base peak, i.e. the most abundant peak, was selected as target ion and when necessary, qualifier ions were used to help identification process (Table 3 ).

\subsection{Processing steps}

\subsubsection{Data pre-processing}

All the data sets were pre-processed in an attempt to reduce the variability of the data induced by the natural intra-variability of fingermark 
Table 3

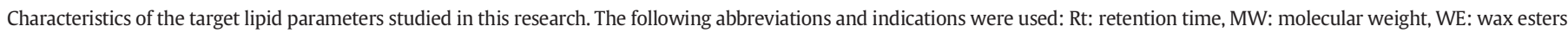

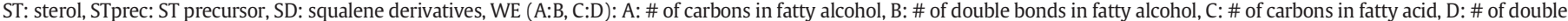
bonds in fatty acid.

\begin{tabular}{|c|c|c|c|c|c|}
\hline Rt (min) & Aging parameters & Abbr. & MW & Targets (qualifiers) & Type \\
\hline 11.822 & Isopropyl dodecanoate & IPD & 242 & $60(102,201)$ & WE \\
\hline 14.189 & 5,9,13-Trimethyl-tetradeca-4,8,12-triene-al & SD1 & 248 & 69 & SD \\
\hline 19.437 & 4,8,13,17,21-Tetramethyl-octadeca-4,8,12,16,20-pentaene-al & SD2 & 316 & 69 & SD \\
\hline 25.419 & 4,9,13,17-Tetramethyl-octadeca-4,8,12,16-tetraeneal & SD3 & 384 & 69 & SD \\
\hline 26.053 & Squalene & SQUAL & 410 & 69 & STprec \\
\hline 29.447 & Cholesterol & CHOL & 386 & $145(368,386)$ & ST \\
\hline 29.594 & Myristyl palmitoleate & MPO & 450 & 236 & WE \\
\hline 29.854 & Myristyl palmitate & MP & 452 & 257 & WE \\
\hline 32.088 & Palmityl palmitoleate & PPO & 478 & 236 & WE \\
\hline 32.348 & Palmityl palmitate & PP & 480 & 257 & WE \\
\hline 33.088 & Wax ester 1 (WE [17:0, 16:1]) & WE1 & 492 & 236 & WE \\
\hline 34.802 & Stearyl palmitoleate & SPO & 506 & 236 & WE \\
\hline 37.663 & Wax ester 2 (WE $[20: 0,16: 1])$ & WE2 & 534 & 236 & WE \\
\hline
\end{tabular}

specimens $[12,15,17,20]$ and by the preparation and analysis steps [26]. Six basic pre-processing combinations often encountered in forensic literature were tested $[20,27-34]$ : normalisation to the internal standard (NormIS), normalisation to the sum of all targets except squalene (NormSum) $)^{1}$, NormIS + standardisation (STD), NormIS + square root (sq.root), NormSum + STD and NormSum + sq.root. It was observed that the best pre-processing method was NormSum + sq.root. Furthermore, it was also tested if all target lipids were useful in the sum. As good results were actually obtained using only IPD, SD1, SD2, SD3, CHOL, MPO and MP, only these compounds were included in the sum of the selected pre-processing (Eq. (1)).

NormSum + sq.root $=\sqrt{\frac{P A_{T C_{i}}}{\sum P A(I P D, \text { SD1-3, CHOL, MPO, MP })}}$

where $P A=$ peak area and $T C_{i}=$ target lipid $i$. It is interesting to highlight here that this aging parameter is mass invariant, i.e. independent of the amount of compounds sampled.

\subsubsection{Chemometrics}

Using the software The Unscrambler ${ }^{\circledR}$ X (version 10.3, Camo ASA, Oslo, Norway) and Origin $₫ 8.1$ (Academic), several statistical treatments were carried out on the pre-processed data sets (Table 4).

PCA is a commonly used multivariate unsupervised classification chemometric technique. It allows reducing the dimension of multivariable data sets through the calculation of new latent variables called principal components (PCs), which are linear combinations of the original variables [35]. Thus, PCA allows detecting sample groups and studying their spread along the different PCs. By studying the coefficients of the linear combinations associated with each PC (i.e. loading plots), the variables influencing the observed separations can also be identified. Furthermore, PCA can be combined with Soft Independent Modelling of Class Analogy (SIMCA) in order to predict the membership of a new sample (a questioned fingermark) to one class (age). When conducting SIMCA, a general PCA has first to be performed on the entire data set in order to identify sample classes. Then, new PCAs are computed for each identified sample class. Finally, by comparing the residual variance of a new sample to the average residual variance of the samples used to make up the different classes, it is possible to obtain a direct measure of the similarity of the new sample to one class or another. Validation processes of PCA and SIMCA were conducted by internal cross-validation in this study [26].

\footnotetext{
1 Squalene was the most abundant compounds in fingermark residue. Thus, in order to avoid its influence to significantly affect the sum, it had to be removed from it. This process is also applied in drug profiling, where the major compounds are often excluded from the sum [30].
}

Exponential regressions based on one aging parameter (univariate statistics) were already reported as fitting the aging of forensic traces, as for examples gunshot residue [23], ink [21,30] and fingermarks left and aged in controlled conditions [17]. In this study, the choice was made to test two exponential regressions: simple exponential regression (Expdec1 in Origin ${ }^{\circledR}$ ) and exponential linear combination regression (Explinear in Origin ${ }^{\circledR}$ ). Overall better results were obtained using the Explinear function and only this regression will thus be shown in the results (Eq. (2)). The parameter $\mathrm{p}_{1}$ corresponds to the offset and $1 / \mathrm{p}_{2}$ to the slope of the exponential part of the curve, while the parameter $p_{3}$ is the offset and $p_{4}$ the slope of the linear part of the curve. These regression parameters and their respective errors can furnish an age estimation of a questioned fingermark, based on its y-value and confidence intervals $[8,9]$, for example at $99 \%$. The lower and upper limits of the confidence interval can be used in order to furnish respectively a minimal and/or maximal age, depending on which confidence interval is crossed by the $y$-value of the questioned fingermark. It is important to note that in this study, the $99 \%$ confidence intervals (usually called confidence bands when applied to regression analysis) were computed using the standard deviation involving residuals, as described by Miller (p. 114-117 of [22]). Furthermore, as stated above, two fingermark specimens were analysed per age, resulting in two aging parameter values (based on Eq. (1)). The average of these values was calculated and reported in the results, as well as their deviation standard representing the error bars visible in the regression figures.

$y=p_{1} e^{-x / p_{2}}+p_{3}+p_{4} x$

PLSR is a frequently used multivariate supervised chemometric technique, well adapted to multivariate and highly correlated datasets [36]. While similar to PCA, the main difference is that the dependant variables Y (in this study, the ages) are known. PLSR models simultaneously both the $\mathrm{X}$ - and $\mathrm{Y}$-matrices to find new X-variables (independent or predictive variables) that will best predict the Y-variables, using linear transformations. These new latent variables are generally called PLSR components [26]. The root mean square errors (RMSE) and the coefficients of determination $\left(\mathrm{R}^{2}\right)$ for calibration and prediction allow

Table 4

Statistics applied on the different data sets collected in this study.

\begin{tabular}{ll}
\hline \# sets & Applied statistics \\
\hline 1-3 known factors & PCA and univariate exponential linear regression \\
4-7 unknown factors & PCA, univariate exponential linear regression and PLSR \\
8 test fingermarks & Age estimation applying models from data set \#7 and \\
& using: \\
& SIMCA based on PCA models \\
& Regression parameters (univariate and PLSR) \\
& LR calculation based on a Bayesian network \\
\hline
\end{tabular}


evaluation of the robustness of the models. Such models can be used to predict the ages of new samples and the prediction errors are computed using the standard equations for $\mathrm{x}$ error calculation of linear regressions [24]. PLS validation was conducted using internal cross-validation [26].

From a legal perspective, the use of a probabilistic approach allows the comparison of two alternative temporal hypotheses by evaluating the probability of a result (value of the aging parameter, E) under two hypotheses called $\mathrm{Hp}$ (prosecution hypothesis) and Hd (defence hypothesis). The ratio between these two probabilities is called likelihood ratio (LR) and quantitatively determines under which hypothesis the result is more probable [37,38] (Eq. (3)):

$\mathrm{LR}=\frac{\mathrm{P}(\mathrm{E} H \mathrm{HP})}{\mathrm{P}(\mathrm{E} H \mathrm{HD})}$

When the data follow a normal distribution, the mean $(\mu)$ and standard deviation $\left(\sigma^{2}\right)$ can be calculated to serve as estimators in the calculation of LRs. These parameters can also be used to build Bayesian networks allowing obtaining the (posterior) probabilities of different pairs of alternative hypotheses taking influence factors and/or measurement errors into account. LR values can also be obtained from such networks, which make them powerful tools for forensic applications $[39,40]$. A Bayesian network was previously reported for age estimations of living persons [41] and could be particularly interesting for fingermark dating applications.

Thus, in this study, a preliminary Bayesian network was built in order to evaluate two alternative temporal hypotheses using fingermark samples exposed to different influence factors, after having tested the normality of the data using the Kolmogorov-Smirnov test [24]. This network is a basic example of the possibilities that such graphical probabilistic tool can offer to estimate the age of fingermarks. More information about the construction of such networks can be found in detailed references on the subject [38-40,42]

\section{Results and discussion}

\subsection{Selection of aging parameters}

As mentioned above, 13 aging variables were selected for this study. Univariate analyses (i.e. univariate exponential regression and LR calculation) were thus conducted separately for each parameter. However, it was noted that the best results were obtained using the pre-processed SQUAL. This is probably due to the fact that this variable was the most abundant in all analysed residue, which allowed more detailed observations to be made. Thus, this results' section will only present the univariate statistics obtained using the pre-processed variable SQUAL.

Concerning the multivariate analyses (i.e. PCA and PLSR), all the 13 pre-processed lipids (i.e. aging variables) were firstly computed to build the models. However, the loading plots indicated that SQUAL was the only variable influencing the results. This was due to the fact that its value was larger than all the other variables. As this could lead to biased analyses [25,26,32], a reduction factor of 20 was applied on the SQUAL value after the pre-processing step in order for all the variables to be on the same scale. The scaled models were then computed and the loading plots indicated six parameters having a major impact on the results: IPD, SD1, SD2, SD3, SQUAL and CHOL. The multivariate models were thus computed using only these six variables.

In summary, from the 13 aging parameters firstly selected, the six following were eventually selected and pre-processed: IPD, SD1, SD2, SD3, SQUAL and CHOL. MPO and MP were also used but only for the definition of the sum calculated at the denominator of the applied pre-processing (see Eq. (1)). The five other lipids were all wax esters and the conducted analyses showed that they were not useful for this study. This result may be due to the irregular presence of these compounds in older fingermark specimens, thus introducing variability preventing relevant results from being obtained. It is also possible that these compounds age in a slower rate, thus preventing aging patterns to be observed.

\subsection{Effects of known factors: donors, substrate and enhancement techniques}

Aging models built for casework dating purposes can include the specific values of known factors. Thus, this section focused on investigating the impact of these factors on the selected aging variables using PCA and exponential linear regressions.

\subsubsection{Donors (set \#1)}

In order to evaluate the effects of the donors on the selected aging parameters, fingermarks of the four selected donors were collected and aged following the fixed standard values.

The PCA computed using the selected six variables IPD, SD1, SD2, SD3, SQUAL and CHOL showed that fingermarks were grouped by donors' type but not by age (Fig. 1). In fact, fingermarks from Donors A and $B$ were grouped together in the left superior part of the PCA and could not be differentiated, which can be explained by the fact that they both belong to the same donor category, i.e. "medium-rich". Then, fingermark specimens of Donor C ("medium-poor") formed a separated group in the left inferior part of the PCA, differentiable from Donors A and B based on the PC-2. This PC accounted for 7\% of the total variance and was mainly influenced by SD1, SD2, SD3, SQUAL and CHOL. Finally, samples of Donor D were all gathered together and completely separated from the other groups on PC-1, which accounted for $84 \%$ of the total variance and was mainly influenced by IPD. Thus, the large variability observed on PC- 1 represented the differences in donor type, "medium-rich" and "medium-poor" donors being on the left of the PCA and "poor" donor being on the right. The observations made based on this PCA highlighted that the effects of the donors' type on the selected variables were larger than the aging effects, as no aging patterns were visible based on fingermark specimens aged from 0 to 27 (Donor D), 28 (Donor C) or 33 (Donors A and B) days old.

Using the value of the pre-processed SQUAL (aging parameter), an exponential linear regression could be fitted on the fingermarks of each donor. Fig. 2 illustrated the obtained results for one donor of each type only (Donors A, C and D), in order to facilitate the visualisation (as Donor B curve was similar to Donor A). Aging patterns showed good exponential linear decay for each donor, particularly for Donors A and D $\left(\mathrm{R}^{2}=0.96\right.$ and 0.94 respectively), Donor $C$ presented a slightly poorer fit $\left(R^{2}=0.88\right)$. However, it was observed that the initial value of the

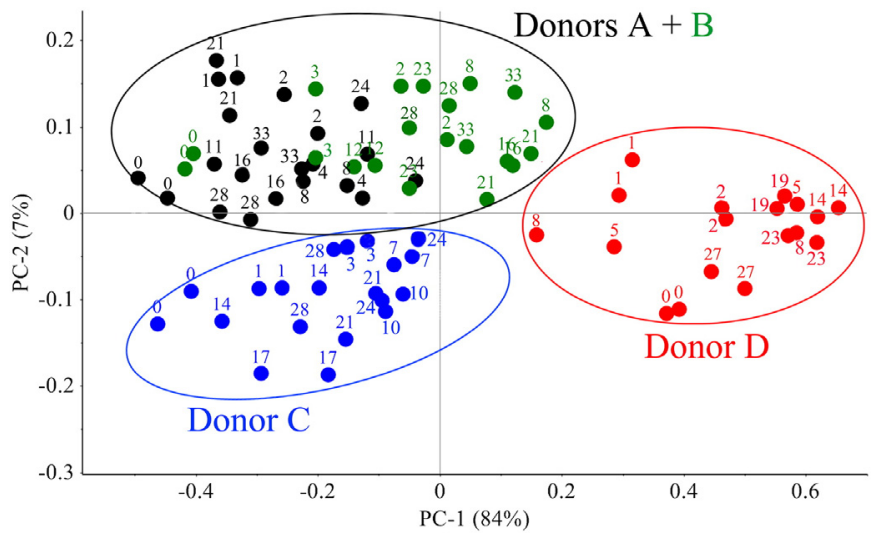

Fig. 1. PCA of fresh and old fingermark specimens from different donors left on filters and aged in the fixed standard conditions. Fingermarks of "medium-rich" Donors A (black) and B (green) were aged from 0 to 33 days, fingermarks of the "medium-poor" Donor $\mathrm{C}$ (blue) were 0 to 28 days old and fingermarks of the "poor" Donor D (red) were aged from 0 to 27 days. Groups were observed based on the type of donor, but not on the age of the fingermarks (the numbers above the points are the fingermark age in days). (For interpretation of the references to colour in this figure legend, the reader is referred to the web version of this article.) 
aging parameter was different between the donors' class: the highest value was obtained in the fresh fingermarks of Donor $\mathrm{A}$, then in those of donor $C$ and the smallest value was observed for Donor D. Furthermore, while the "medium-rich" Donor A and the "medium-poor" Donor $C$ showed aging pattern until 28 days old and above, no more aging were visible after 14 days for the "poor" Donor D. Exponential linear regression thus allowed observing aging patterns but highlighted large differences due to donor, as PCA did.

\subsubsection{Substrate (set \#2)}

The effects of substrates on the selected aging parameters were studied leaving fingermarks on microfiber filters and glass. The deposition and storage conditions of the fingermarks were fixed at the standard values. As similar results concerning substrate effects were obtained for each donor, the results are illustrated in this section using only Donor A.

The PCA computed using the six selected variables IPD, SD1, SD2, SD3, SQUAL and CHOL showed two clear groups corresponding to each substrate, but no age tendency could be observed (Fig. 3). This separation occurred principally on the PC-2, which accounted for $30 \%$ of the total variance and was mainly influenced by SD1, SD2, SD3, SQUAL and CHOL. Thus, all these variables were affected by the substrate differences. IPD principally influenced PC-1 (accounting for $63 \%$ of the total variance) but no clear grouping tendency could be detected on this axis. Thus, the variance observed on PC- 1 was probably due to the uncontrollable intra-variability of fingermark specimens $[12,15]$. The observations made based on this PCA highlighted that the effects of the substrate type on the target variables were larger than the aging effects, as no aging patterns were visible.

Using the value of the pre-processed SQUAL (aging parameter), an exponential linear regression could be fitted based on the fingermarks left on each substrate, as illustrated for Donor A in Fig. 4. Aging patterns were visible for each substrate and showed good exponential linear decay $\left(R^{2}=0.96\right.$ for both substrate). However, it was observed that the initial value of the aging parameter was different between the substrates: a higher value was obtained for fingermarks left on glass than for those left on filters. In order to understand this observation, the raw values of each variable were studied and revealed that the peak

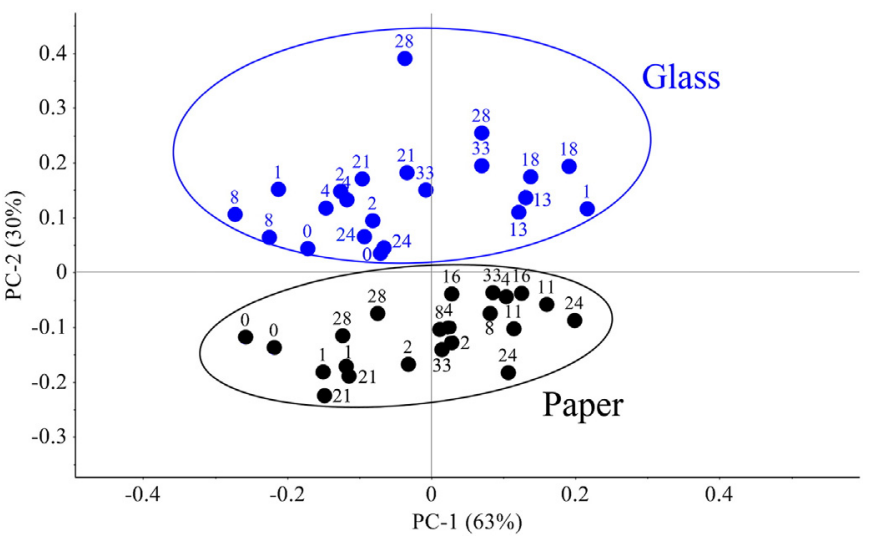

Fig. 3. PCA of fresh and old fingermark specimens from Donor A ("medium-rich"), left on glass (blue) or microfiber filters (black) and aged until 33 days in the fixed standard conditions. Groups observed based on substrate, but not on fingermark age (the numbers above the points are the fingermark age in days). (For interpretation of the references to colour in this figure legend, the reader is referred to the web version of this article.)

areas of IPD, SD1, SD2, SD3, CHOL, MP and PP were more than 10 times smaller in fingermarks left on glass than in fingermarks left on filters. The peak area of squalene was also smaller on glass, but only by a factor of 2. Thus, the value of the aging parameter being obtained by dividing the peak area of squalene by the sum of the peak areas of the other target lipids, its value became higher for glass. It is interesting to note that these results correspond to the literature, as a previous study also reported smaller recovered amounts of squalene and cholesterol from fingermarks left on glass than from those left on paper [17]. While transferred amounts may actually be different on these substrates, the main explanation to this observation probably lies in the different extraction procedures: paper can be directly immerged in the extraction solvents, while glass has to be "swabbed" to extract the lipids.

Furthermore, it is also important to note that the aging decay showed by fingermarks was slightly different between the two studied substrates. In fact, a sharp reduction between 0 and 1 day was observed on paper, while this reduction was softer on glass. This observation did

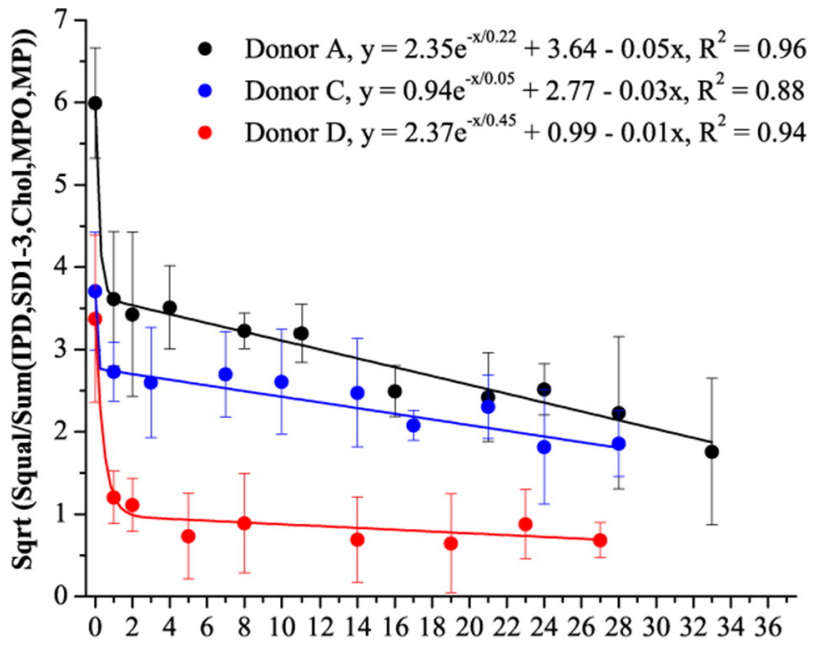

Age (Days)

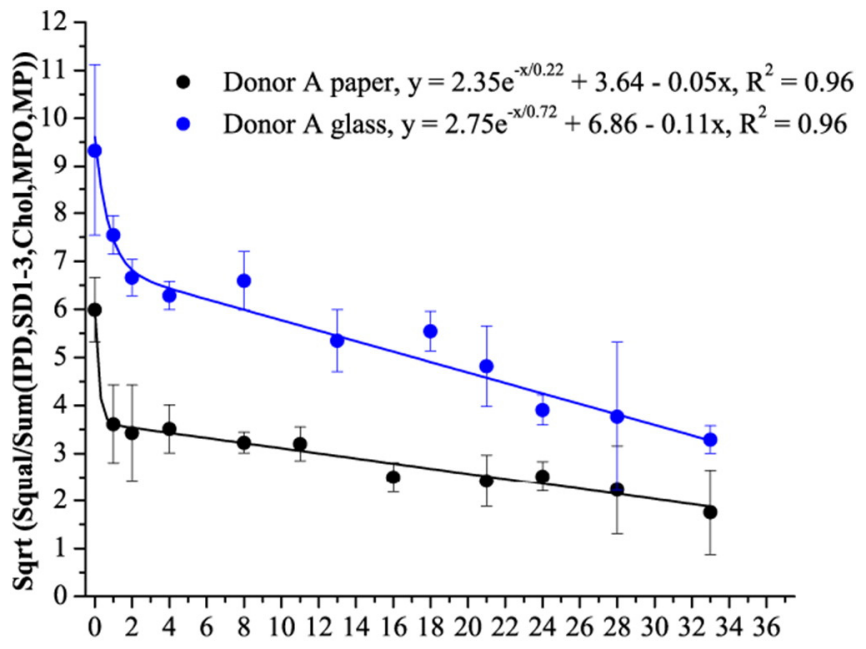

Age (Days)

Fig. 2. Exponential linear regressions of fingermarks from Donor A ("medium-rich", black), C ("medium-poor", blue) and D ("poor", red) left on filters and aged in the fixed standard conditions. The pre-processed SQUAL was used as aging parameter and good fits were visible for each donor. Differences in the initial value of the parameter were due to donors' class and induced different aging dynamics. (For interpretation of the references to colour in this figure legend, the reader is referred to the web version of this article.)

Fig. 4. Exponential linear regressions using fingermarks from Donor A ("medium-rich") left on glass (blue) or microfiber filter (black) and aged in the fixed standard conditions. The pre-processed SQUAL was used as aging parameter and good fits were visible on each substrate. Differences in the initial value of the parameter were observed between substrate and induced different aging dynamics. (For interpretation of the references to colour in this figure legend, the reader is referred to the web version of this article.) 
not correspond to the previous paper mentioned above, as it reported a sharper reduction on glass than on paper [17]. This can be due to the fact that a different aging parameter was used in this previous study, highlighting the importance of the selected aging variables for fingermark aging and dating research [8,9]. Exponential linear regression thus allowed observing aging patterns but also highlighted large differences due to substrate, as did the PCA.

\subsubsection{Enhancement (set \#3)}

The effects of aluminium powder application (Argentorat) on the targeted aging parameters over time were studied using the "medium-poor" Donor C. Fingermarks were left on glass, the deposition conditions were fixed at the standard values and they were left to age at $20^{\circ} \mathrm{C}$ in the dark or exposed to a normal $24 \mathrm{~h}$ cycle. However, as similar results concerning the effect of enhancement were obtained when fingermarks were stored under both lighting conditions, the results are illustrated using only the fingermarks stored in the dark.

The PCA computed using the six selected aging variables IPD, SD1, SD2, SD3, SQUAL and CHOL showed two clear groups corresponding to enhanced and not enhanced fingermarks, but no age tendency could be observed (Fig. 5). This separation occurred principally on the PC-1, which accounted for $68 \%$ of the total variance and was influenced by the six variables. PC- 2 accounted for $14 \%$ of the total variance but no clear grouping tendency could be observed and this variance was probably due to the uncontrollable variability of fingermarks $[12,15]$. The observations made based on this PCA highlighted that the effects of the application of enhancement powder affected the studied aging variables in such a way that aging effects were not visible.

Based on the value of the pre-processed SQUAL (aging parameter), an exponential linear regression could be fitted based on the enhanced and not-enhanced fingermarks (Fig. 6). Aging patterns were visible for powdered and non-powdered fingermarks and showed good exponential linear decay ( $\mathrm{R}^{2}=0.94$ and 0.97 respectively) corresponding to what was observed for fingermarks left on glass (Section 3.2.2). However, it was observed that the initial value of the aging parameter was different: a much higher value was obtained for non-enhanced fingermarks than for enhanced ones, as already highlighted in the literature [19]. This difference in the initial composition was probably due to the filtration steps necessary to analyse enhanced fingermarks and also affected the aging decay. In fact, while the non-enhanced fingermarks showed aging pattern until 36 days, no further aging was visible after 10 days for powder-enhanced fingermarks. Exponential linear

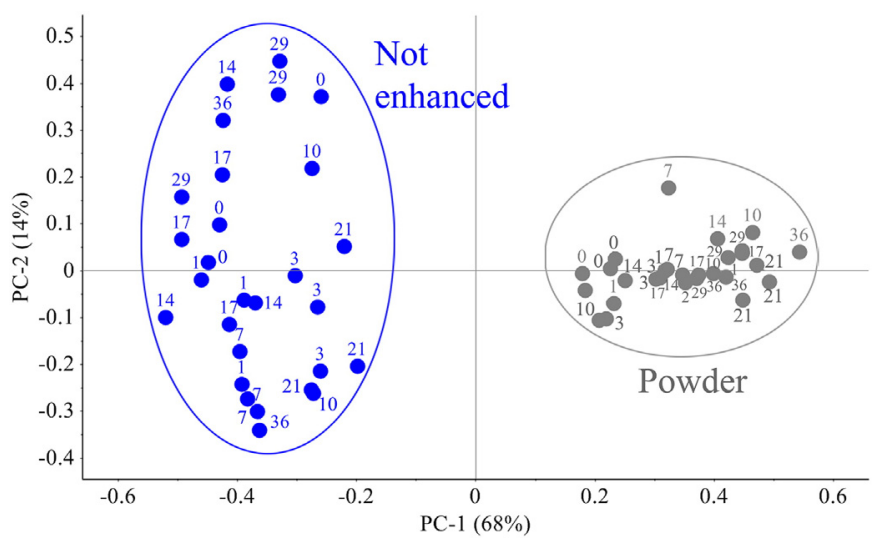

Fig. 5. PCA of fresh and old fingermark specimens from Donor C ("medium-poor") left on glass, aged in the fixed standard conditions and powdered (grey) or not (blue) before analysis. Fingermarks were aged from 0 to 36 days. Groups were observed based on the presence of powder before analysis or not, but not on the age of the fingermarks (the numbers above the points are the fingermark age in days). (For interpretation of the references to colour in this figure legend, the reader is referred to the web version of this article.)

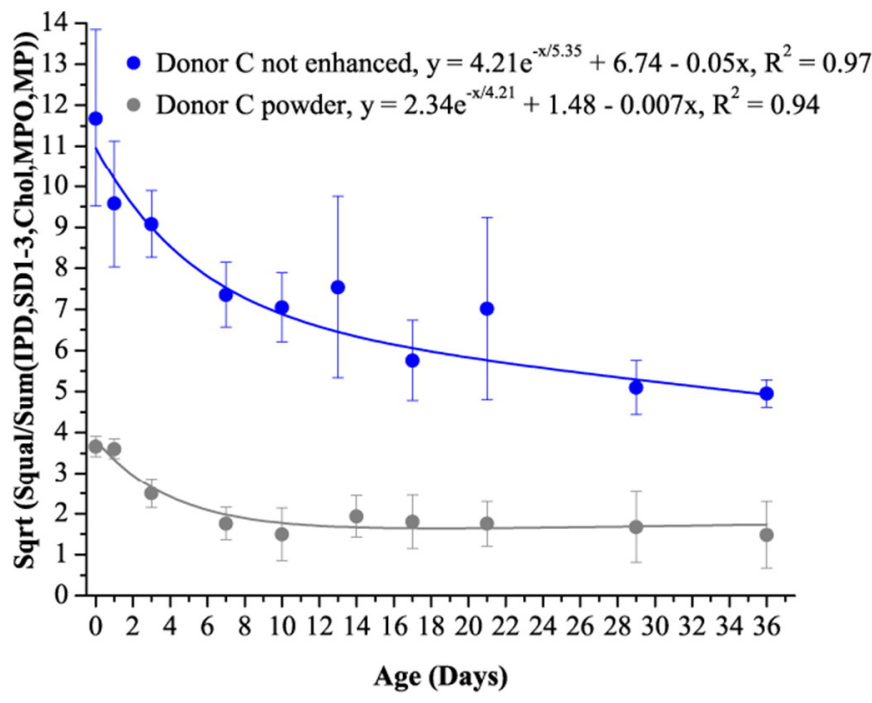

Fig. 6. Exponential linear regressions of fingermarks from Donor C ("medium-poor") left on glass, aged in the fixed standard conditions and powdered (grey) or not (blue) before analysis. The pre-processed SQUAL was used as aging parameter and good fits were visible for both curves. Large differences in the initial values of the aging parameter were observed due to the application of powder and induced different aging dynamics. (For interpretation of the references to colour in this figure legend, the reader is referred to the web version of this article.)

regression thus allowed observing aging patterns but also highlighted large differences due to the application of enhancement powder.

\subsection{Effects of unknown factors: deposition moment and pressure, temperature and lighting conditions}

It is essential that aging models built for casework dating purposes show robustness to the effects of unknown factors. This section investigated the impact of some of these factors on the selected aging variables. The possibility to build robust aging models using chemometrics commonly used in other forensic areas, i.e. PCA, exponential linear regressions and PLSR, was also studied. This allowed evaluating the potential of these modelling techniques for fingermark dating purposes.

\subsubsection{Deposition moment (set \#4)}

The effects of deposition moments, i.e. at which period a fingermark was left, were studied using fingermarks of donor C ("medium-poor") left in April (C1) or July (C2) of the same year. The deposition and storage conditions were fixed at the standard values.

The PCA computed using the six selected variables IPD, SD1, SD2, SD3, SQUAL and CHOL showed an age separation between fingermarks at $0,<10$ and $\geq 10$ days old (Fig. 7 ). The younger fingermarks were mainly grouped in the upper part of the PCA, while the older were in the lower part. This age separation was mainly due to PC-2, which accounted only for $7 \%$ of the total variance. This means that even if the variability due to age was visible, it represented a very small part of the data set variance. PC-2 was influenced by SD1, SD2 and SD3; the squalene derivatives were thus responsible for the visible aging groups. Far more variability was accounted by the PC-1 (87\%), which was influenced by IPD, SQUAL and CHOL. It was responsible for a separation corresponding to the moment at which the fingermarks were left. In fact, the fingermarks left in April (C1) were mainly grouped on the left of the PCA, while July samples $(\mathrm{C} 2)$ were gathered together on the right. Only the 0 day old samples of C1 and C2 were grouped together on the left. This observation did not mean that no differences existed between fresh fingermarks left at different deposition moments, but that these differences were less important than the differences due to aging. Thus, the PCA presented in Fig. 7 highlighted that the deposition 


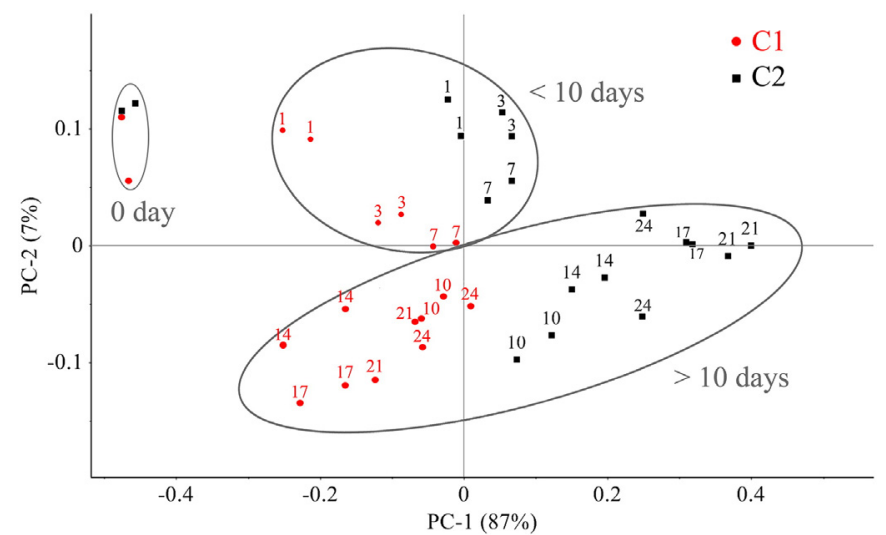

Fig. 7. PCA of fresh and old fingermark specimens from Donor C ("medium-poor") left on microfiber filters in April ( $\mathrm{C} 1$, red circles) or in July ( $\mathrm{C} 2$, black squares) and stored in the fixed standard conditions. Fingermarks were aged from 0 to 24 days. Three groups based on fingermark age were visible: $0,<10$ or $\geq 10$ days old (the numbers above the points are the fingermark age in days). However, a separation due to deposition moment was also visible, as C1 was mainly grouped on the right and C2 on the left of this PCA. (For interpretation of the references to colour in this figure legend, the reader is referred to the web version of this article.)

moment affected the selected variables, but that this influence factor still allowed the observation of aging effects.

Based on the value of the pre-processed SQUAL (aging parameter), an exponential linear regression could be fitted on each set ( $\mathrm{C} 1$ and C2) as illustrated in Fig. 8A. These regressions showed very good fits $\left(\mathrm{R}^{2}=0.94\right.$ and 0.99 respectively $)$ and similar aging decays were observed on each curve. However, the initial value of the aging parameter was different for each deposition moment and affected the aging dynamics. Exponential linear regression thus allowed observing aging patterns, as PCA did, but also highlighted differences in the initial value of the parameter due to the deposition moment, which was not visible with PCA. Furthermore, in order to study the possibility of building a relevant aging model combining both tested deposition moments (as this factor would not be precisely known in caseworks), an exponential linear regression was fitted using C1 and C2 fingermarks together (Fig. 8B). In order to build this figure $\mathrm{B}$, the aging parameter values obtained for each age in both curves of figure $A$ were averaged and the standard deviation was also calculated over these values. All B-figures of this study were built in the same way.

An aging decay showing a good fit $\left(\mathrm{R}^{2}=0.95\right)$ was observed, but large standard deviations were visible for each age. The calculated

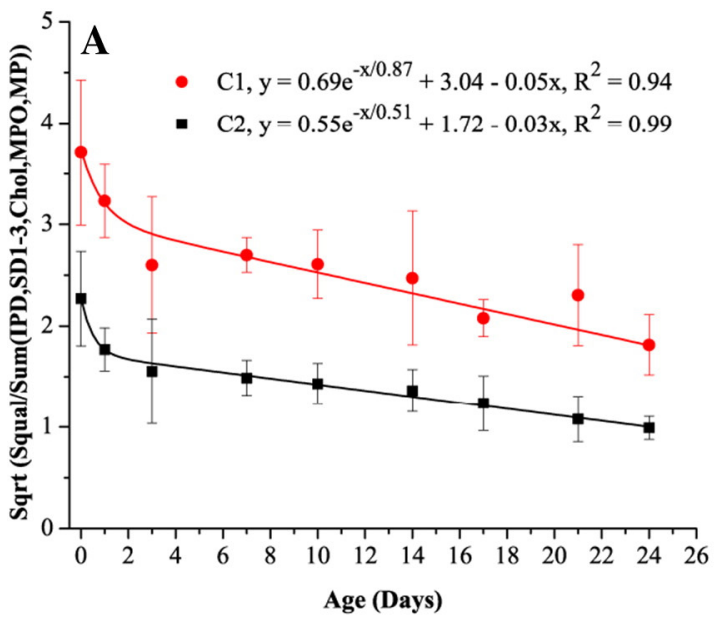

confidence intervals at $99 \%$ were large, principally because of these large deviations. These results highlighted the importance to consider the deposition moment as significant influence factor while developing fingermark dating methodologies based on univariate regressions.

Finally, PLSR was computed using the six selected variables IPD, SD1, SD2, SD3, SQUAL and CHOL (Fig. 9). The obtained model showed good linearity $\left(R^{2}>0.8\right)$ and the root mean square errors of calibration (RMSEC) and validation (RMSEV), expressing the general model fitting, were good (respectively \pm 3.11 days and \pm 3.71 days). Fingermarks of 0 , 1 and 3 days old could not be separated from each other's but were completely differentiated from fingermarks of 10 days old and older. On the contrary, fingermarks of 7 days old showed a larger spread and could be included in younger or older fingermarks. This model can thus reliably differentiate $\leq 3$ or $\geq 10$ days old fingermarks. This result is similar to the observations made using PCA (Fig. 7) and highlighted the capacity of PLSR to model aging in a promising way, even when fingermarks were left at different deposition moments.

\subsubsection{Pressure of deposition (set \#5)}

The effects of the deposition pressure on the selected aging parameters were studied using fingermarks of Donor D ("poor") left at 100, 500 or $1000 \pm 50 \mathrm{~g}$. The other deposition and storage conditions were fixed at the standard values.

The PCA computed using the six selected variables IPD, SD1, SD2, SD3, SQUAL and CHOL showed an age separation between $\leq 8$ and $>8$ days old fingermarks (Fig. 10). The younger fingermarks were visible in the right part of the PCA, while the older ones were grouped in the left part. Thus, this age separation mainly happened on PC-1 (accounting for $84 \%$ of the total variance) and was due to all the six variables. However, it should be noted that the younger fingermarks were very widely spread, particularly those at 0 days. As this spreading was not due to pressure, this observation is most likely due to the uncontrollable initial intra-variability of the fingermark specimens $[12,15]$. PC-2 accounted for $11 \%$ of the total variance but no relevant grouping was observed on this axis. This variability was thus probably again due to the uncontrollable intra-variability of fingermark specimens. Furthermore, no groups based on the deposition pressure were observed in this PCA. Thus, the deposition pressure did not seem to significantly affect the selected variables using PCA, as groups could be formed based on fingermark age but not based on the applied pressure.

Based on the value of the pre-processed SQUAL, an exponential linear regression could be fitted on each pressure, as illustrated in Fig. 11A. The 500 and $1000 \mathrm{~g}$ regressions showed good fits $\left(R^{2}=0.94\right.$ and 0.98 respectively), while the $100 \mathrm{~g}$ regression was poorer $\left(\mathrm{R}^{2}=\right.$

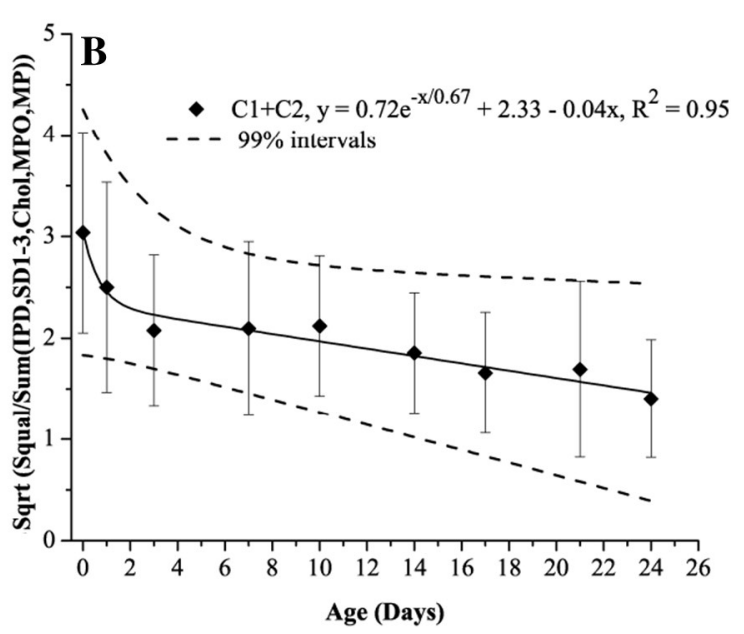

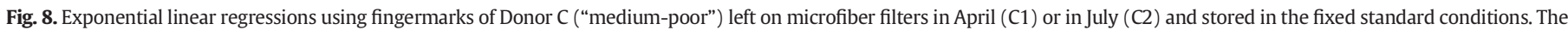

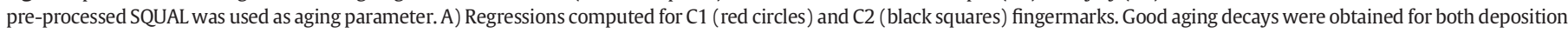

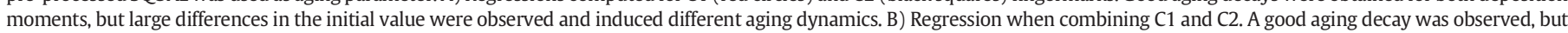
large standard deviations were visible. (For interpretation of the references to colour in this figure legend, the reader is referred to the web version of this article.) 


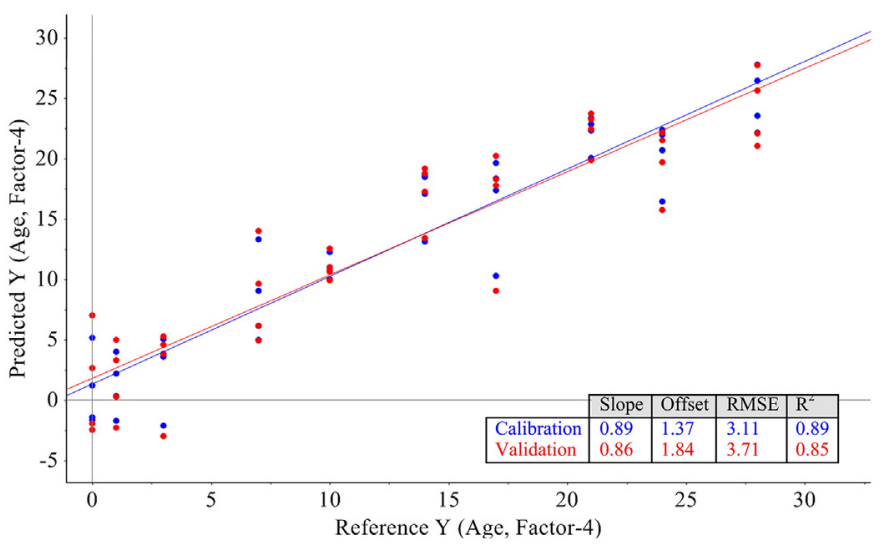

Fig. 9. PLS regression using fingermarks of Donor C ("medium-poor") left on filters in April or in July and stored in the fixed standard conditions. Calibration (Cal, blue) and crossvalidation (Val, red) points were calculated and good fits were obtained. Fingermarks of 0,1 and 3 days old could be differentiated from those of 10 days old and older. However, significant spreading due to different deposition moments was visible. (For interpretation of the references to colour in this figure legend, the reader is referred to the web version of this article.)

0.75). Furthermore, differences in the initial value of the aging parameter due to the different pressures were also noted and affected the aging decay. In fact, aging was visible until around 18 days for $1000 \mathrm{~g}$ fingermarks, while aging tendencies stopped after around 14 days for $500 \mathrm{~g}$ and 5 days for $100 \mathrm{~g}$ fingermarks respectively. Exponential linear regression thus allowed aging patterns to be observed, as PCA did, but also highlighted differences in the initial value of the aging parameter due to the deposition pressure, which was not the case using PCA. Furthermore, in order to study the possibility of building a relevant aging model combining the three tested deposition pressures (as this factor would not be precisely known in caseworks), an exponential linear regression was fitted using 100, 500 and $1000 \mathrm{~g}$ fingermarks together (Fig. 11B). An aging decay showing a good fit $\left(\mathrm{R}^{2}=0.95\right)$ was observed, but only until around 4 days, because the curve flattened after this point. This is likely due to the type of donor used ("poor"). In fact, as this donor left little material in the fresh fingermark residue, aging probably became difficult to observe, because compound amount diminished over time. Relatively small standard deviations were visible for each age, and the calculated confidence intervals at 99\% included them all.
Pressure is thus not a factor largely influencing the creation of fingermark aging regression model, which is a positive result for the development of fingermark dating methodologies based on univariate regressions.

Finally, PLSR was computed using the six selected variables IPD, SD1, SD2, SD3, SQUAL and CHOL (Fig. 12). The obtained model showed a very poor fit for calibration and validation $\left(R^{2}=0.52\right.$ and 0.48 , respectively) and large spreading was visible for each age. The root mean square errors of calibration (RMSEC) and validation (RMSEV), were relatively high (respectively \pm 6.72 days and \pm 7.13 days). Because of the large spreading observed at each age, fingermark ages could not be differentiated. These results are poorer than the separation obtained by PCA and the regression obtained using an univariate exponential linear function. This highlighted that PLSR did not show good robustness to model aging when very different pressures were applied.

\subsubsection{Temperature conditions (set \#6)}

The effects of storage temperature on the selected aging parameters were studied using fingermarks stored at 15,20 or $25^{\circ} \mathrm{C}$. These samples were left at the standard pressure on glass or microfiber filters and aged in the dark. As similar observations were made concerning temperature effects between different donors and substrates, this section illustrates the results using fingermarks of Donor B left on microfiber filters.

The PCA was conducted using the six selected variables IPD, SD1, SD2, SD3, SQUAL and CHOL (Fig. 13). The fresh fingermarks $(t=0)$ were not included in this model because their values prevented the proper visualisation of age and/or temperature groups. An age separation was detectable for fingermarks stored at 15 and $20^{\circ} \mathrm{C}$. In fact, the fingermarks up to 3 days old were visible in the lower left part of the PCA, while the older ones were grouped in the upper part (fingermarks above 3 days old). This separation occurred on PC-2, which accounted for $10 \%$ of the total explained variance and was influenced by $\mathrm{CHOL}$ and SQUAL. Concerning fingermarks stored at $25^{\circ} \mathrm{C}$, no separation based on age was visible and all specimens were gathered together in the lower right part of the PCA. Thus, this analysis highlighted that storage temperature should be carefully considered when dealing with fingermark dating purposes. In fact, aging patterns were visible for fingermarks stored at 15 and $20^{\circ} \mathrm{C}$, but not for those stored at $25^{\circ} \mathrm{C}$. This temperature probably induced a higher variability among the targeted variables.

Based on the value of the pre-processed SQUAL, an exponential linear regression could be fitted for each temperature (Fig. 14A). These

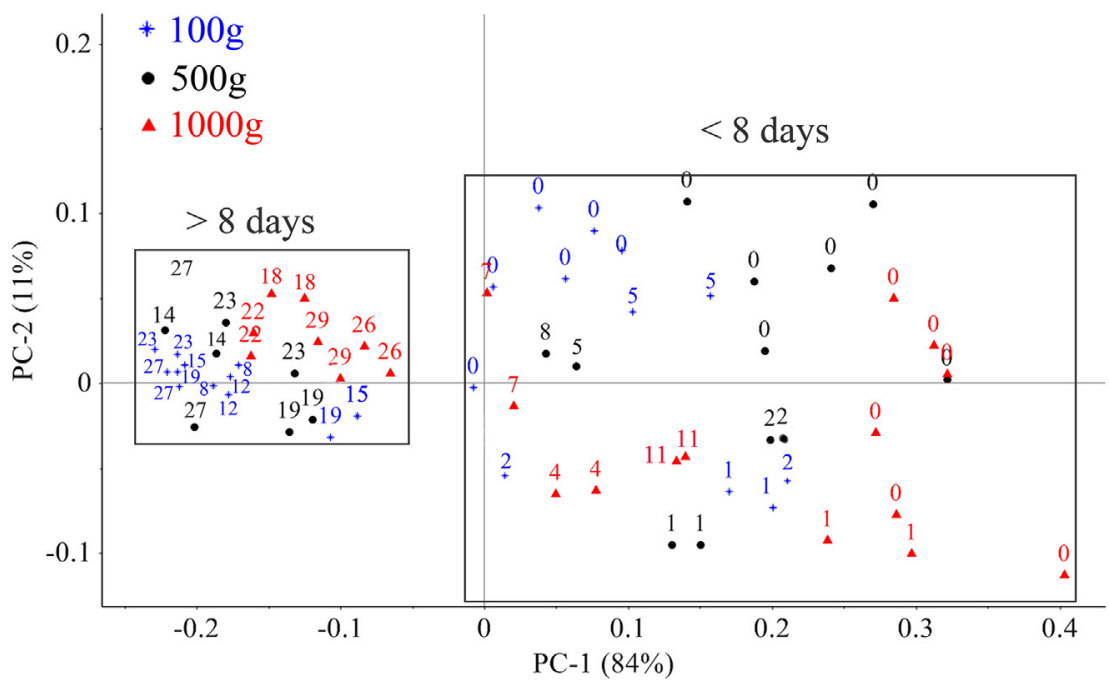

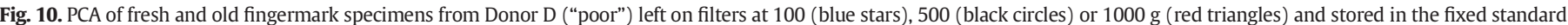

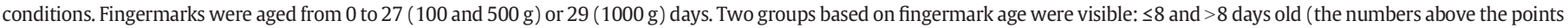

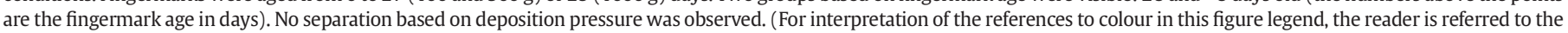
web version of this article.) 

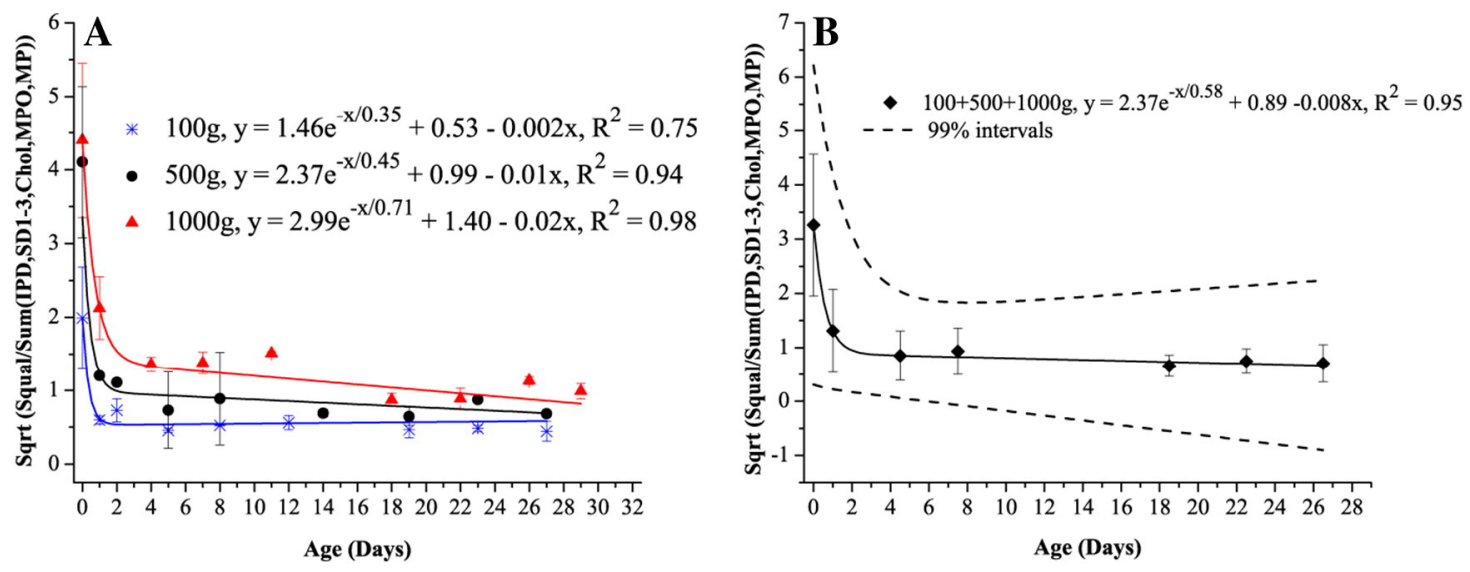

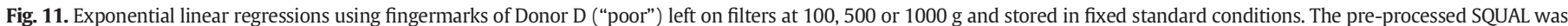

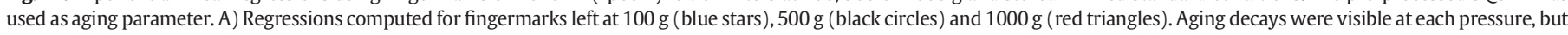

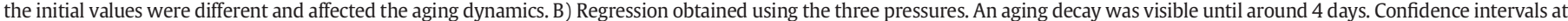
$99 \%$ included the standard deviations. (For interpretation of the references to colour in this figure legend, the reader is referred to the web version of this article.)

regressions showed very good fits $\left(\mathrm{R}^{2}=0.98\right.$ for 15 and $20^{\circ} \mathrm{C}$ and 0.94 for $25^{\circ} \mathrm{C}$ ), but differences in aging dynamics were observed. In fact, the $15{ }^{\circ} \mathrm{C}$ curve had a sharper slope in its exponential part and the $25^{\circ} \mathrm{C}$ regression showed a far quicker decay than the other curves. Differences were also observed in the initial value of the aging parameter, corresponding to the variability caused by different deposition moments (see Section 3.3.1). Thus, exponential linear regressions allowed observing aging patterns for each temperature, which was not the case using PCA. It also highlighted that the storage temperature caused complex effects on the kinetics of the selected aging parameter (particularly for $25^{\circ} \mathrm{C}$ ), which, this time, corresponded to PCA results. Furthermore, in order to study the possibility to build a relevant aging model combining the three tested storage temperatures (as this factor could be difficult to precisely know in casework), an exponential linear regression was fitted using 15, 20 and $25{ }^{\circ} \mathrm{C}$ fingermarks together (Fig. 14B). Fingermark specimens of $0,1,16,21$ and 28 days old were analysed for each temperature and were thus used to build this general curve. A very good aging decay was observed $\left(R^{2}=0.99\right)$. The calculated confidence intervals at $99 \%$ were calculated and included the standard deviation of each age. Temperature seems thus not a factor largely influencing the creation of fingermark aging regression model, which is a positive result for the development of fingermark dating methodologies based on univariate regressions.

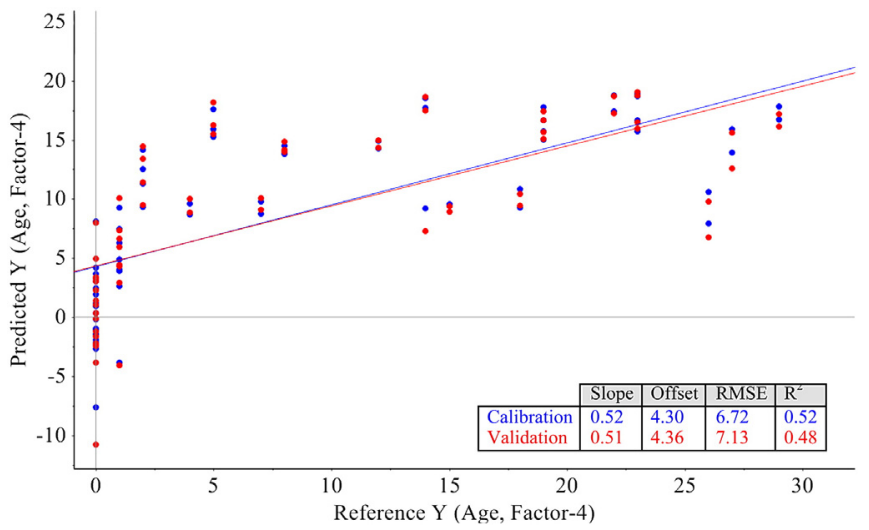

Fig. 12. PLS regression using fingermarks of Donor D ("poor") left on filters at 100, 500 or $1000 \mathrm{~g}$ and stored in the fixed standard conditions. Calibration (Cal, blue) and crossvalidation (Val, red) points were calculated and poor fits were obtained $\left(R^{2}=0.52\right.$ and 0.48 , respectively). Significant spreading due to difference in deposition pressures was visible for each age and no age could be clearly separated from another. (For interpretation of the references to colour in this figure legend, the reader is referred to the web version of this article.)
Finally, PLSR was computed using the six selected variables IPD, SD1, SD2, SD3, SQUAL and CHOL (Fig. 15). However, the obtained model showed a very poor fit for calibration and validation $\left(\mathrm{R}^{2}=0.53\right.$ and 0.41 , respectively). Large spreading was visible for each age. The root mean square errors of calibration (RMSEC) and validation (RMSEV) corresponded to about 7 days, which is relatively high as the older fingermarks were 35 days. Because of the large spreading observed at each age, only fingermarks of 0 day old could be completely separated from fingermarks of 12 days old and above. Thus, in this study, PLSR did not show a good robustness to model the age of fingermark stored under different temperatures.

\subsubsection{Lighting conditions (set \#7)}

The effects of lighting conditions on the selected aging parameters were studied based on the fingermarks of Donor C ("medium-poor") stored in the dark or exposed to a normal $24 \mathrm{~h}$ cycle (called light hereafter), at standard temperature. The fingermarks were left on glass at the standard pressure and some were enhanced before analysis. Similar observations were made concerning lighting effects based on enhanced or not enhanced fingermarks. Thus, this section illustrated the results using only non-enhanced specimens.

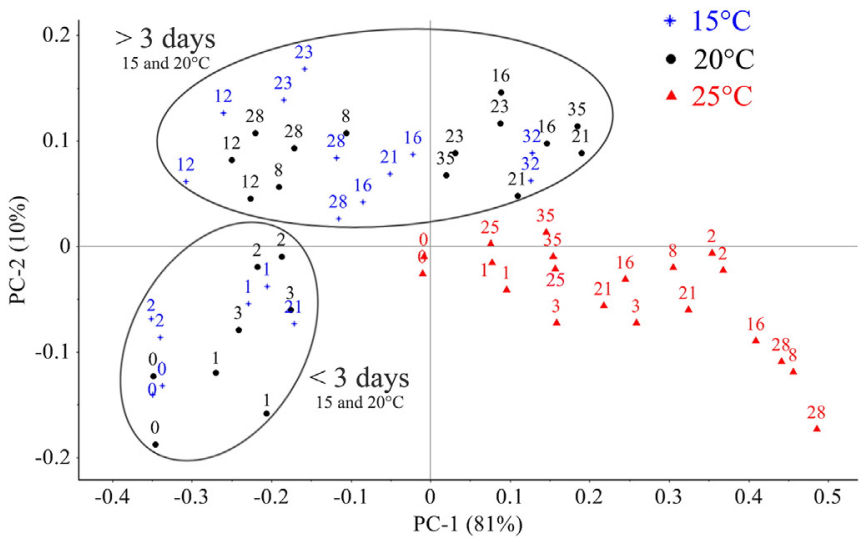

Fig. 13. PCA of aged fingermark specimens from Donor B ("medium-rich") left on microfiber filters and stored at 15 (blue stars), 20 (black circles) or $25^{\circ} \mathrm{C}$ (red triangles). Fingermarks were 1 to $32\left(15^{\circ} \mathrm{C}\right)$ or $35\left(20\right.$ and $\left.25^{\circ} \mathrm{C}\right)$ days old. Two age groups were visible for 15 and $20{ }^{\circ} \mathrm{C}: \leq 3$ and $>3$ days old (the numbers above the points are the fingermark age in days). Fingermarks stored at $25^{\circ} \mathrm{C}$ showed no age separation. (For interpretation of the references to colour in this figure legend, the reader is referred to the web version of this article.) 

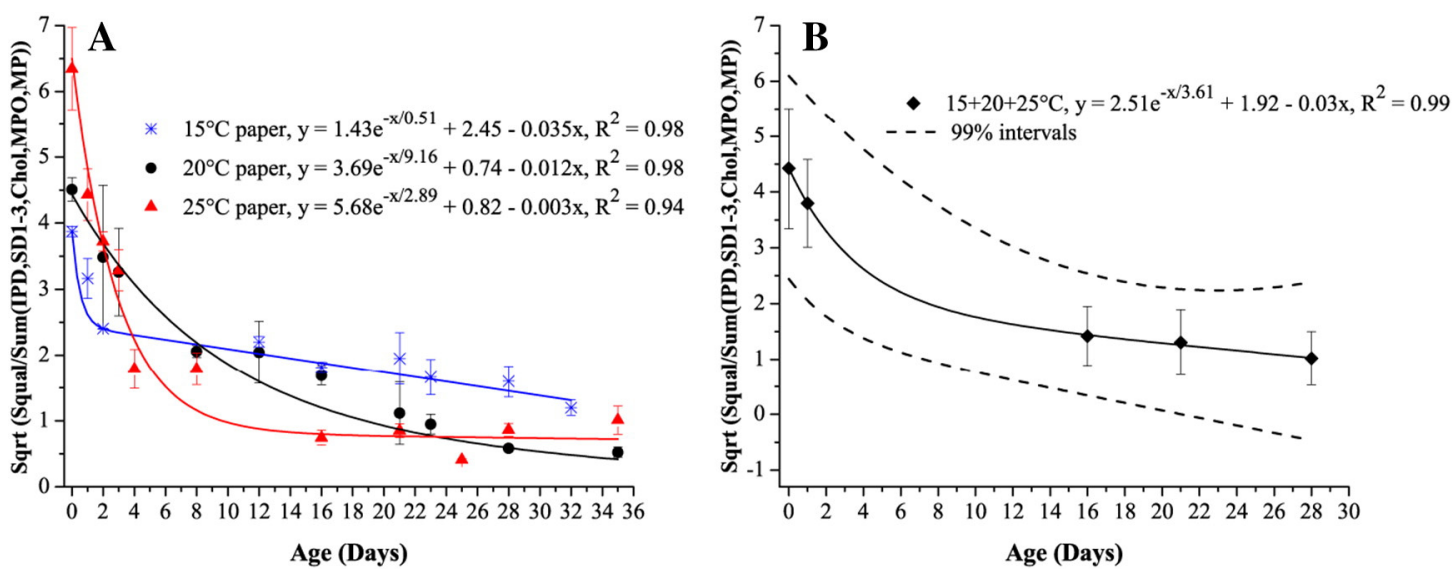

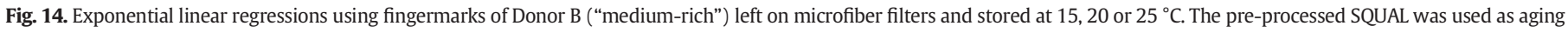

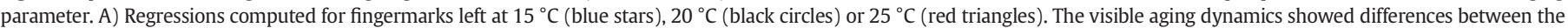

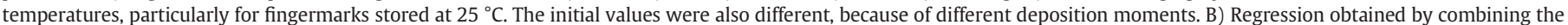

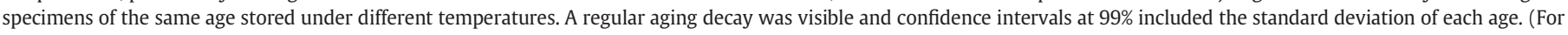
interpretation of the references to colour in this figure legend, the reader is referred to the web version of this article.)

The PCA computed using the six selected variables IPD, SD1, SD2, SD3, SQUAL and CHOL firstly showed a clear separation between fingermarks stored in the dark and those stored in the light (Fig. 16). This separation was mainly visible on PC-1, which accounted for $49 \%$ of the total variance and was influenced by SD2, SQUAL and CHOL. However, an age separation between $<10$ and $\geq 10$ days old fingermarks was also visible on the PC-2, older specimens being mainly in the upper part of the PCA and younger in the lower part. PC-2 accounted for $23 \%$ of the total variance and was mainly influenced by IPD, SD1 and SD3. The storage in the dark or in the light clearly affected the selected aging parameters using PCA, but still allowed the formation of groups based on fingermark ages.

Based on the value of the pre-processed SQUAL, an exponential linear regression could be fitted for each lighting condition (Fig. 17A). These regressions showed very good fits $\left(R^{2}=0.99\right.$ and 0.97 , for storage in the light and dark, respectively), but differences in aging dynamics were observed. In fact, the values of the aging parameter of fingermarks stored in the light decreased much more rapidly than those for fingermarks stored in the dark. A clear aging pattern was visible until 36 days for fingermarks stored in the dark, while fingermarks stored in the light seemed to show no more aging tendency after 14 to

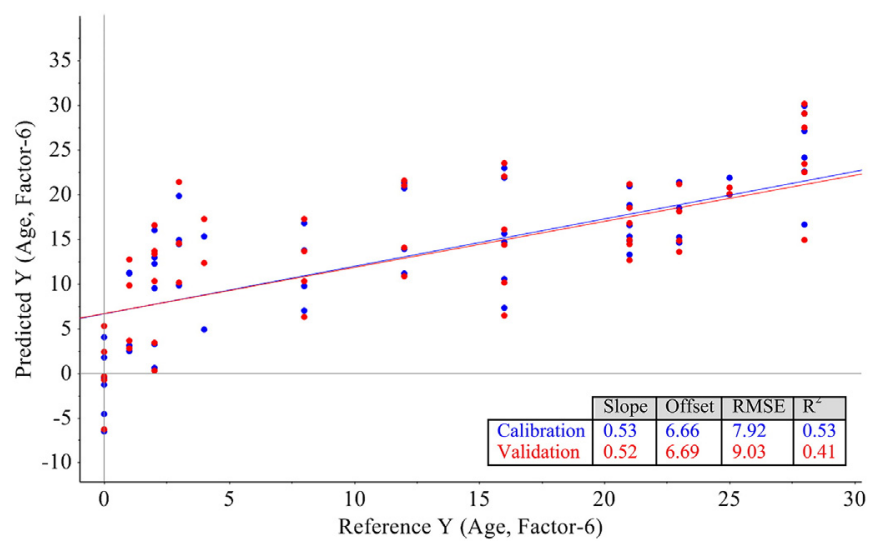

Fig. 15. PLS regression using fingermarks of Donor B ("medium rich") left on microfiber filters and stored at 15,20 or $25^{\circ} \mathrm{C}$. Calibration (Cal, blue) and cross-validation (Val, red) points were calculated. Poor fits were obtained $\left(R^{2}=0.53\right.$ and 0.41 , respectively). Significant spreading due to different storage temperatures was visible for each age. Only fingermarks of 0 day old could be completely separated from fingermarks of 12 days old and above. (For interpretation of the references to colour in this figure legend, the reader is referred to the web version of this article.)
17 days. Furthermore, fingermarks stored in the dark showed more variability at each age than fingermarks stored in the light. This is probably because in the light, the initial composition differences between specimens are rapidly masked by the large aging effects, while in the dark, this intra-variability is still visible, as aging is rather slower. Thus, these observations highlighted the capacity of exponential linear regression to observe aging patterns for fingermarks stored in the light or in the dark. Furthermore, it also indicated that the kinetics of the selected aging parameter was influenced differently by the different lighting conditions.

In order to study the possibility of building a relevant aging model combining the two tested lighting conditions (as this factor would not be precisely known in casework), an exponential linear regression was fitted on fingermarks stored in the light and in the dark (Fig. 17B). Very good aging decay was observed $\left(R^{2}=0.99\right)$ until 36 days, but the standard deviations calculated for each age were significant. Thus, the calculated confidence intervals at $99 \%$ were very large. As this model showed an aging dynamic, it could be used for the estimation of the age of a questioned mark, but a very large uncertainty should be expected. This highlights the importance to consider the lighting conditions as a potentially limiting factor while developing fingermark dating methodologies based on univariate regressions.

Finally, PLSR was computed using the six selected variables IPD, SD1, SD2, SD3, SQUAL and CHOL (Fig. 18). However, the obtained model showed a poor fit for calibration and validation $\left(R^{2}=0.58\right.$ and 0.48 , respectively) and large spreading was visible for each age. The root mean square errors of calibration (RMSEC) and validation (RMSEV), expressing the general model fitting, were relatively high (respectively \pm 6.70 and \pm 7.78 days). Because of the large spreading observed at each age, only fingermarks of 0 and 1 day old could be separated from specimens of 29 days old and older. Thus, in this study, PLSR did not show a good robustness to model the age of fingermark stored under different lighting conditions.

\subsection{Blind tests (set \#8): age estimation based on models developed using set \#7}

In order to evaluate the potential of aging modelling for fingermark dating, blind tests were conducted with eight test fingermarks (FM1-8) left at $500 \mathrm{~g}$ on glass by Donor C ("medium-poor"). The responsible investigator was made aware of the age of these fingermarks and of the applied storage conditions only after having conducted the GC/MS analyses and age estimations. The models built using the non-enhanced fingermarks of set \#7 stored in the dark or in the light (Section 3.3.4) 


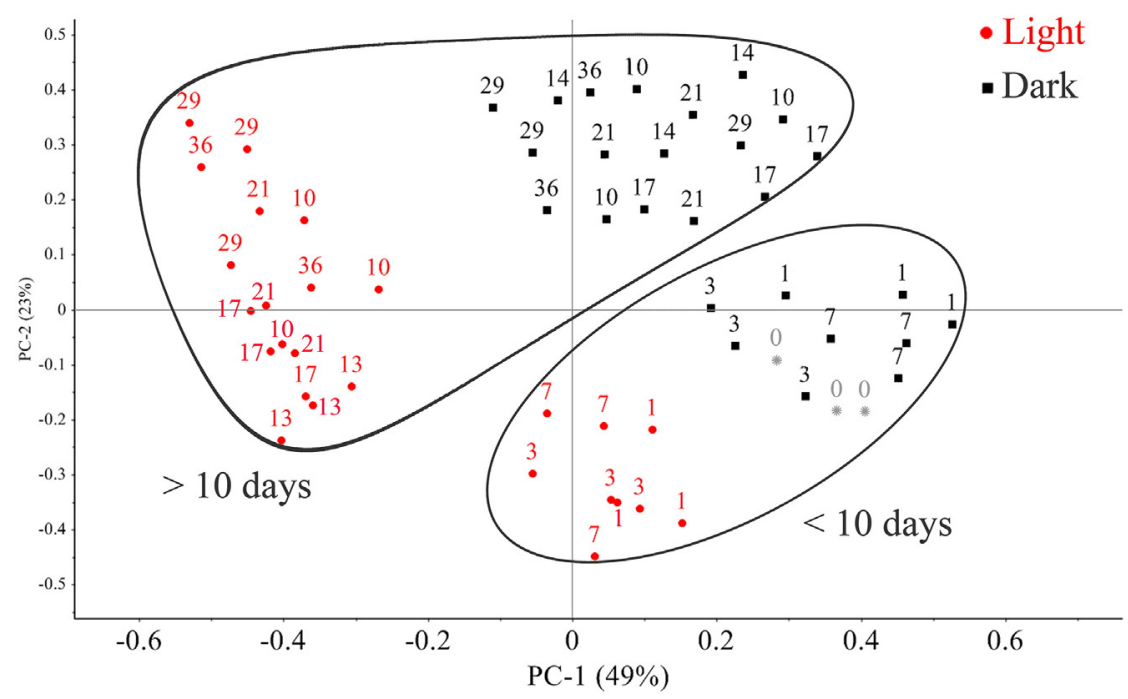

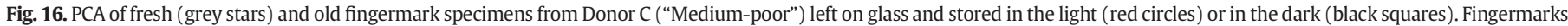

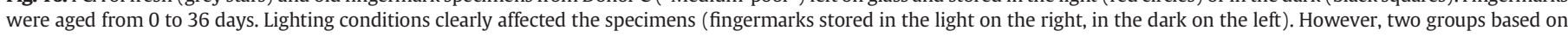

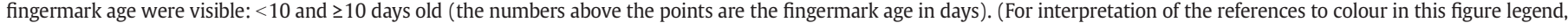
the reader is referred to the web version of this article.)

were used because the known influence factors corresponded to the test fingermarks. The models grouping the specimens stored in both lighting conditions were used, as this factor is unknown in the practice. Age estimations were firstly conducted using classification statistics (SIMCA based on PCA classes) and univariate (exponential linear function) or multivariate (PLS) regressions. Then, a probabilistic approach using a Bayesian network for the calculation of likelihood ratios (LR) was also tested.

\subsubsection{Age estimations using classification statistics and regressions}

Based on the PCA presented in Section 3.3.4 (Fig. 16), an age classification was observed between $<10$ and $\geq 10$ days old fingermarks, regardless of the tested lighting conditions. Thus, two new PCAs were computed for each identified sample class and SIMCA was then applied. By comparing the residual variance of the test fingermarks to the average residual variance of the samples used to make up the different classes, it was possible to obtain a direct measure of the similarity of the test samples to the $<10$ or $\geq 10$ days old class. Five of the eight test fingermarks were correctly classified; FM3-6 and FM8 (Table 5). One fingermark could not be classified (FM1) because its residual variance exceeded the upper limit for every modelled class. This fingermark was 22 days old and had been stored next to a window in an open box. The incapacity of the model to classify this fingermark could be due to the fact that the $>10$ days old class was largely spread, highlighting a high variability. Furthermore, two fingermarks (FM2 and 7) were classified in the wrong group. In fact, these fingermarks were respectively 34 and 22 days old and stored in a closed drawer, but they were classified in the $<10$ days old group. This could be due to the fact that even if a separation was visible between $<10$ and $\geq 10$ days old fingermarks stored in the dark, these two classes were very close, which could lead to a wrong classification. These observations highlighted that while PCA and SIMCA demonstrated encouraging results concerning fingermark age classification, more samples should be included in the models in order to better define the variability of each class and increase the robustness of the model. Furthermore, these results tend to highlight that the aging of fingermarks in the dark is more variable than in the light, probably because it is slower and still affected by the initial variability of fresh fingermarks.

The exponential linear regression models with $99 \%$ confidence intervals obtained for fingermarks stored in both lighting conditions were
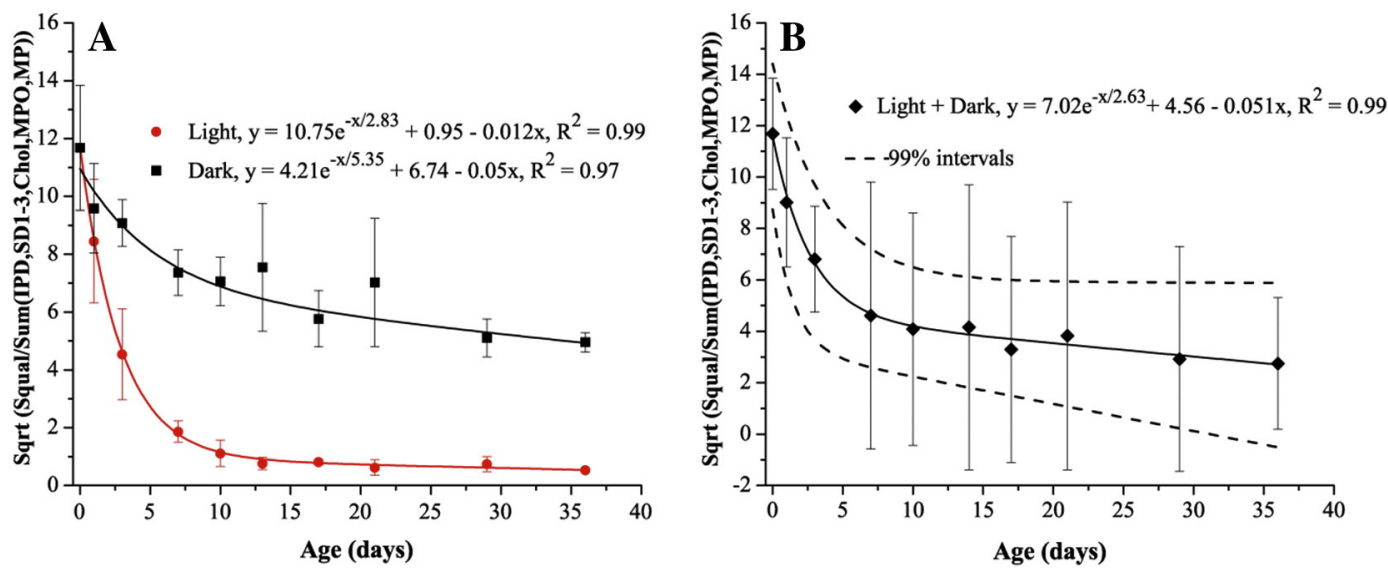

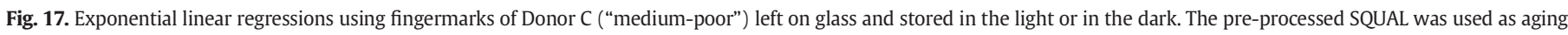

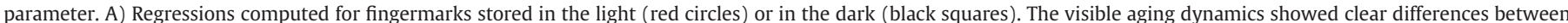

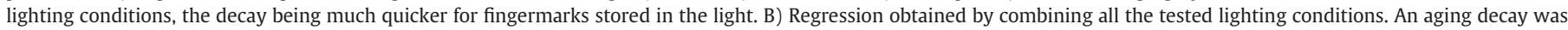

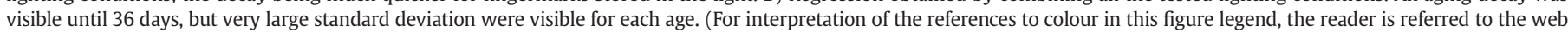
version of this article.) 


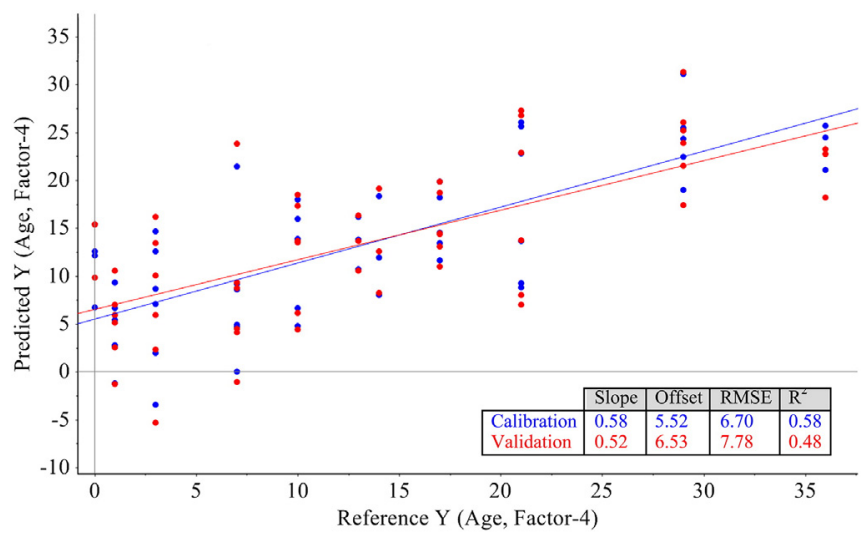

Fig. 18. PLS regression using fingermark of Donor C ("Medium-poor") left on glass and stored in the light or in the dark. Calibration (Cal, blue) and cross-validation (Val, red) points were calculated and poor fits were obtained $\left(R^{2}=0.58\right.$ and 0.48 , respectively). Significant spreading was visible for each age and only fingermarks of 0 and 1 day old could be separated from fingermarks of 29 days and above. (For interpretation of the references to colour in this figure legend, the reader is referred to the web version of this article.)

used to estimate the age of the test fingermarks (Fig. 17B). These estimations yielded a minimal or maximal age, depending if the aging parameter value crossed the lower or upper limit of the confidence interval. For example, for a low value crossing the lower limit of confidence, it was concluded that the fingermark was equal or older than the age given by the lower limit (i.e. minimal age). On the contrary, if the obtained value crossed the upper limit, it was estimated that the fingermark was equal or younger than the age given by the upper limit (i.e. maximal age). The age of the test fingermarks 1-5 was correctly evaluated, while wrong estimations were obtained for FM6-8 (Table 5). It is important to note that some estimations were correct but very large; for example, FM2 was estimated to be $\geq 3$ days old and this fingermark was actually 34 days old. The estimations obtained for FM6-8 were incorrect, probably because of the large variability observed in the middle of the regression curve, corresponding to the real ages of these fingermarks (i.e. 8 and 22 days old). Overall, the univariate regression gave interesting results, but the age estimations were not very precise. Thus, larger sample size should be examined in order to determine if better results could be obtained.

The parameters of the PLSR model obtained using fingermarks stored in the light and in the dark (Fig. 18) were used to estimate the age of the test fingermarks and the associated error. The age of FM1, FM3, FM5-6 and FM8 was correctly estimated, while incorrect estimations were obtained for FM2, 4 and 7. The correct estimations were obtained along with an error of around 8 days (the oldest fingermarks being 36 days old). The incorrectly estimated fingermarks were the oldest ones ( 22 and 34 days old). This can be explained by the fact that the PLSR model showed particularly large variance at around 20 days, thus likely causing wrong estimations. Furthermore, the fit of

\section{Table 5}

Real age, storage conditions and age estimations [in days] of the eight test fingermarks (FM). The estimations were conducted using three models: SIMCA ( $<10$ or $\geq 10$ days old), univariate regression (minimal or maximal age based on $99 \%$ confidence intervals) or PLS regression (absolute age and errors). Correct estimations were shaded in light green and wrong results in dark red. N.A.: not assigned.

\begin{tabular}{|l|r|c|c|c|c|}
\hline FM & Age & $\begin{array}{c}\text { Storage } \\
\text { in an office }\end{array}$ & SIMCA & $\begin{array}{c}\text { Univariate regression } \\
\text { Conf. interval 99\% }\end{array}$ & PLS regression \\
\hline 1 & 22 & Window & N.A. & $\geq 22$ & $16.2 \pm 8.4$ \\
\hline 2 & 34 & Drawer & $<10$ & $\geq 3$ & $12.1 \pm 9.4$ \\
\hline 3 & 1 & Window & $<10$ & $\leq 2$ & $6.6 \pm 7.4$ \\
\hline 4 & 34 & Window & $\geq 10$ & $\geq 23$ & $21.1 \pm 6.3$ \\
\hline 5 & 1 & Drawer & $<10$ & $\leq 1$ & $5.8 \pm 9.4$ \\
\hline 6 & 8 & Drawer & $<10$ & $\leq 2$ & $5.9 \pm 8.9$ \\
\hline 7 & 22 & Drawer & $<10$ & $\leq 4$ & $6.1 \pm 7.3$ \\
\hline 8 & 8 & Window & $<10$ & $\leq 6$ & \\
\hline
\end{tabular}

the PLSR was very poor ( $R^{2} 0.58$ for calibration and 0.48 for validation), which could also explain that this model gave correct estimations with relatively large errors along with wrong estimations. It should be further studied whether more robust results and smaller errors could be obtained by including more specimens in PLSR models.

\subsubsection{Age estimations using a probabilistic approach}

The age of the eight test fingermarks was estimated using LRs calculation based on a Bayesian network. In order to show the feasibility of such an approach and its potential for fingermark dating issues, a pair of temporal hypotheses was evaluated. These hypotheses were chosen based on the ages of the collected comparison fingermarks:

- Hp (prosecutor hypothesis): the fingermark is less than 10 days old
(age $\leq 10$ days)
- Hd (defence hypothesis): the fingermark is more than 14 days old (age $\geq 14$ days).

The pre-processed squalene was chosen as aging parameter. Its value was calculated for comparison fingermarks that were stored in the dark in a drawer (three replicates) or exposed to a circadian cycle next to a window (three replicates) over different time periods. As these data were normal based on the Kolmogorov-Smirnov test, its means $(\mu)$ and standard deviations $\left(\sigma^{2}\right)$ were calculated for each age group and each storage condition (Table 6 ), i.e. for fingermarks stored in a drawer ( $\mu_{\text {dark }}$ et $\sigma_{\text {dark }}^{2}$ ) and for fingermarks stored next to a window $\left(\mu_{\text {ight }}\right.$ et $\sigma^{2}$ light $)$. These data were used to build a Bayesian network based on the three following discrete nodes (Fig. 19):

- "Hp/Hd": represents the two temporal hypotheses mentioned above - "Ages": represents the different ages of the comparison fingermarks

- "Storage": represents the storage conditions of the comparison fingermarks, i.e. in the dark (drawer) or exposed to a circadian cycle (window).

All the mutually exclusive states of these nodes were fixed equiprobable. Furthermore, a fourth continuous node was created, i.e. the node "SQUAL", which contains the means and standard deviations of the pre-processed squalene value (aging parameter) obtained for comparison fingermarks (Table 6). The (directed) arrows that connect the nodes together show the existing probabilistic dependence relationships between them. Thus, the node "Squalene" is dependant of the nodes "Storage" and "Ages", as the lighting conditions and the age of the fingermarks will affect the obtained value of the pre-processed squalene. Furthermore, the node "Hp/Hd" is dependant of the node "Ages", as the actual age of the questioned fingermark will define which temporal hypothesis is supported.

For simplicity, the prior probabilities for each hypothesis were fixed each at $50 \%$. Thus, when the value of the pre-processed squalene of the test fingermarks (E, Eq. (3)) was inserted in the node "SQUAL", the posterior probabilities of this $\mathrm{E}$ value under the two tested hypotheses could be obtained through $\mathrm{P}(\mathrm{E} \mid \mathrm{H})$. These probabilities were then used to calculate the LR values (based on Eq. (3)).

The state of the node "Storage" was first set equiprobable in order to calculate LRs based on comparison fingermarks aged in unknown storage conditions $\left(\mathrm{LR}_{\mathrm{tot}}\right)$. Then, the node "Storage" was set on "Drawer" and the LR values for the test fingermarks stored in the dark were calculated $\left(\mathrm{LR}_{\mathrm{dark}}\right)$. Finally, the state "Window" was selected and the LR values for the test fingermarks exposed to a circadian cycle were calculated $\left(\mathrm{LR}_{\text {light }}\right)$. In a potential real application, $\mathrm{LR}_{\text {dark }}$ or $\mathrm{LR}_{\text {light }}$ could be computed only if the storage conditions of the questioned fingermarks were known.

The obtained results (Table 7) highlighted that the values of $\mathrm{LR}_{\text {tot }}$ (storage conditions unknown) supported the correct hypothesis for six out of eight test fingermarks. The two errors were done on the test 
Table 6

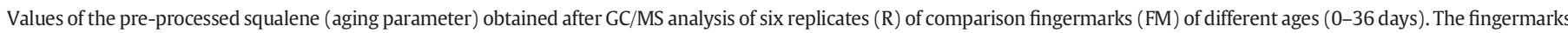

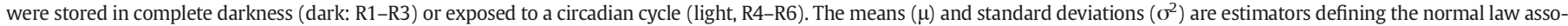
ciated with these data.

\begin{tabular}{|c|c|c|c|c|c|c|c|c|c|c|}
\hline \multirow[t]{2}{*}{ Age of the comparison FM [days] } & \multicolumn{6}{|c|}{ Pre-processed squalene values } & \multicolumn{4}{|l|}{ Estimators } \\
\hline & $\mathrm{R} 1$ & $\mathrm{R} 2$ & R3 & $\mathrm{R} 4$ & R5 & $\mathrm{R} 6$ & $\mu_{\text {dark } \_R 1-R 3}$ & $\sigma_{\text {dark_R1-R3 }}^{2}$ & $\mu_{\text {light_R4-R6 }}$ & $\overline{\sigma_{\text {light_R4-R6 }}^{2}}$ \\
\hline 0 & 12.7 & 8.4 & 10.9 & 12.7 & 8.4 & 10.9 & 10.68 & 4.69 & 10.68 & 4.69 \\
\hline 1 & 10.4 & 11.8 & 13.5 & 10.9 & 6.9 & 7.5 & 11.88 & 2.38 & 8.45 & 4.57 \\
\hline 3 & 8.6 & 7.2 & 7.3 & 5.3 & 5.5 & 2.7 & 7.70 & 0.65 & 4.54 & 2.48 \\
\hline 7 & 12.1 & 10.5 & 8.5 & 1.4 & 1.4 & 0.6 & 10.36 & 3.20 & 1.11 & 0.21 \\
\hline 10 & 9.3 & 11.0 & 9.9 & 2.2 & 1.4 & 2.0 & 10.06 & 0.71 & 1.87 & 0.14 \\
\hline 14 & 8.4 & 12.8 & 10.4 & 0.5 & 0.9 & 0.9 & 10.55 & 4.88 & 0.76 & 0.04 \\
\hline 17 & 9.0 & 7.7 & 9.6 & 0.9 & 0.8 & 0.7 & 8.76 & 0.95 & 0.82 & 0.01 \\
\hline 21 & 8.7 & 10.2 & 11.1 & 0.9 & 0.4 & 0.5 & 10.02 & 1.48 & 0.62 & 0.07 \\
\hline 29 & 6.1 & 11.1 & 7.2 & 1.0 & 0.6 & 0.6 & 8.10 & 7.06 & 0.74 & 0.07 \\
\hline 36 & 5.2 & n.a & 4.7 & 0.5 & n.a. & 0.5 & 4.96 & 8.24 & 0.54 & 0.10 \\
\hline
\end{tabular}

fingermarks 2 and 7, aged in a drawer for respectively 34 and 22 days but evaluated as being $\leq 10$ days old. Globally, the values of $\mathrm{LR}_{\text {tot }}$ were very close to 1 and thus only yielded weak support that would be considered as inconclusive in a potential real application. Only the older fingermarks stored next to a window and exposed to light (test fingermark 1 and 4) allowed obtaining significant $\mathrm{LR}_{\text {tot }}$. This was probably due to the degradation processes occurring more quickly in the light than in the dark and confirmed previous observations made using the SIMCA model.

When the storage conditions were taken into account $\left(\mathrm{LR}_{\text {dark }}\right.$ and $\left.L_{\text {light }}\right)$, no erroneous results were obtained. While the fingermarks stored in the dark mainly yielded LR values close to 1 (weak support), fingermarks stored in the light allowed obtaining LR strongly supporting the correct hypothesis. This observation highlighted again that the aging process of fingermarks exposed to light was more rapid than in the dark, thus allowing the formation of clearer aging pattern and the calculation of more significant LR values.

These results also clearly showed that storage conditions should be known in order for the proposed probabilistic model to yield correct and significant supports.

This preliminary probabilistic model gave promising results and proved the feasibility of such an approach for fingermark dating issues. However, the obtained results also highlighted that storage conditions (i.e., exposition to light) largely affected fingermarks aging and should be known to correctly infer fingemarks ages. Thus, fingermark aging should be further studied on larger data population in order to evaluate if an optimisation of the proposed probabilistic approach is possible. This optimisation could for example include more aging parameters and more influence factors (multivariate probabilistic approach).

\section{Discussion and conclusion}

This study investigated the effects of influence factors on aging parameters based on selected lipids analysed in fingermarks over one month using GC/MS. Out of 13 selected lipids, the following six were eventually targeted to follow aging: isopropyl dodecanoate (IPD), squalene (SQUAL), 3 squalene derivatives (SD1, 2 and 3) and cholesterol (CHOL). These compounds were pre-processed using normalisation to the sum followed by the square root in order to reduce specimen variability. The values of myristyl palmitoleate (MPO) and myristyl palmitate (MP) were added to calculate the sum. The six pre-processed variables were used as aging parameters in the construction of multivariate models (i.e. PCA and PLSR), while univariate models were built (i.e. exponential linear regression and LR calculations) using only the pre-processed SQUAL.

This study first evaluated the effects of known influence factors on aging parameters (i.e. different donors, substrates and fingermark enhancement). It was highlighted that these factors significantly affected the initial value of the selected aging parameters and also had an impact on their aging dynamics. In fact, fingermarks were mainly classified based on the values of these influence factors, thus largely preventing relevant age classification using PCA. Exponential linear regressions highlighted that the "poorer" the donor was (in lipid composition), the lower were the values of the selected aging parameter and the shorter were the periods during which aging tendencies were detectable. These observations thus clearly demonstrated that the known factors brought large variability to fingermark initial composition and aging. Therefore, aging models built for fingermarks dating perspective using the targeted aging parameters should be based on comparison

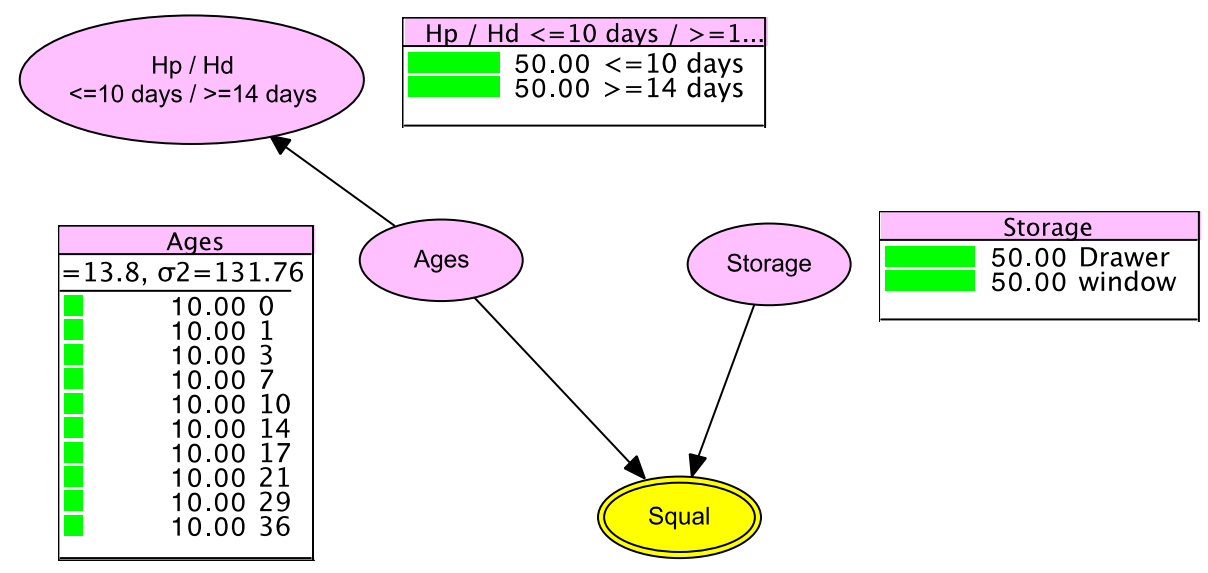

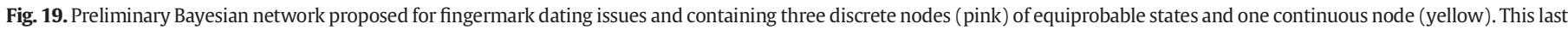

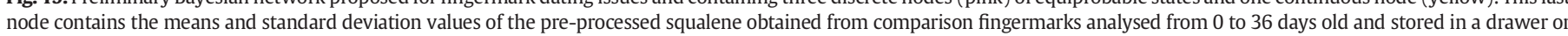
next to a window. (For interpretation of the references to colour in this figure legend, the reader is referred to the web version of this article.) 
Table 7

Calculation of likelihood ratios (LRs) based on a Bayesian network using the value of the pre-processed squalene (aging parameter). The LRs were calculated using comparison fingermarks stored in all tested storage conditions ( $\left.L R_{\text {tot }}\right)$, or by setting the storage conditions as known in order to use only the fingermarks stored in a drawer $\left(\mathrm{LR}_{\text {dark }}\right)$ or next to a window ( $\mathrm{LR}_{\text {light }}$ ). $\mathrm{H}$ gives the supported hypotheses: $\mathrm{Hp} \leq 10$ or $\mathrm{Hd} \geq 14$ days old. The results highlighted in green supported the right hypothesis, while those highlighted in red yielded a wrong result.

\begin{tabular}{|c|c|c|c|c|c|c|c|c|c|}
\hline \multirow[t]{2}{*}{ \# Test FM } & \multirow{2}{*}{$\begin{array}{l}\text { Actual age } \\
\text { (days) }\end{array}$} & \multirow[t]{2}{*}{ Storage } & \multirow{2}{*}{$\begin{array}{c}\text { Aging } \\
\text { parameter }\end{array}$} & \multicolumn{2}{|c|}{$\mathrm{LR}_{\mathrm{tot}}$} & \multicolumn{2}{|c|}{$\mathrm{LR}_{\text {dark }}$} & \multicolumn{2}{|c|}{$\mathrm{LR}_{\text {light }}$} \\
\hline & & & & Value & $\mathrm{H}$ & Value & $\mathrm{H}$ & Value & $\mathrm{H}$ \\
\hline 1 & 22 & Window & 0.87 & 0.097 & $\geq 14$ & 1 & 1 & 0.097 & $\geq 14$ \\
\hline 3 & 1 & Window & 11.23 & 2.208 & $\leq 10$ & 1 & 1 & $2.87 \mathrm{E}+247$ & $\leq 10$ \\
\hline 4 & 34 & Window & 0.81 & 0.078 & $\geq 14$ & 1 & 1 & 0.079 & $\geq 14$ \\
\hline 8 & 8 & Window & 7.91 & 1.430 & $\leq 10$ & 1 & 1 & $2.02 \mathrm{E}+117$ & $\leq 10$ \\
\hline 2 & 34 & Drawer & 3.35 & 1.353 & $\leq 10$ & 0.005 & $\geq 14$ & 1 & 1 \\
\hline 5 & 1 & Drawer & 13.39 & 3.634 & $\leq 10$ & 2.740 & $\leq 10$ & 1 & 1 \\
\hline 6 & 8 & Drawer & 12.09 & 2.778 & $\leq 10$ & 2.077 & $\leq 10$ & 1 & 1 \\
\hline 7 & 22 & Drawer & 8.85 & 1.043 & $\leq 10$ & 0.707 & $\geq 14$ & 1 & 1 \\
\hline
\end{tabular}

fingermarks of a given donor (identified with the questioned mark), left on a comparable substrate and enhanced with the same technique than the one used for the questioned mark, in order to avoid these sources of variability.

The effects of unknown influence factors (i.e. deposition pressure, deposition moment, temperature and light conditions) were then evaluated using PCA, exponential linear regression and PLSR in order to study the robustness of aging models for dating purposes. PCA was able to group fingermarks based on their age, mainly separating specimens under 8 or 10 days old from older ones, except when different storage temperatures were applied. In fact, fingermarks stored at $15^{\circ} \mathrm{C}$ and $20^{\circ} \mathrm{C}$ showed an age separation between specimens under 3 days old from older ones, but no age separation could be observed at all for specimens stored at $25^{\circ} \mathrm{C}$, as these showed a higher variability.

Exponential linear regressions highlighted aging patterns for each tested influence factors. Good regression curves $\left(R^{2} \geq 0.95\right)$ were obtained even when combining different values of each influence factor (for example, combining fingermarks left at 100, 500 and $1000 \mathrm{~g}$ ). However, the $99 \%$ confidence interval was significantly larger when different lighting conditions were applied, because the regression levelled off much earlier for fingermarks stored in the light than for those stored in the dark.

PLSR gave poor results when different pressures, temperatures and lighting conditions were applied on the fingermark specimens. Large spreading was observed for each age. On the contrary, when different deposition moments were tested, the obtained PLSR model showed good calibration and validation fit parameters. These results highlighted that PLSR was not the better way to model fingermark aging based on the chosen aging parameters when unknown influence factors were applied.

In order to test these modelling techniques in pseudo realistic situation, eight test fingermarks were prepared in controlled conditions (known donor, substrate, enhancement and pressure) but stored in unknown conditions. The age of these fingermarks was evaluated using models built with fingermarks of the same donor, left on the same substrate and unenhanced. SIMCA based on PCA classes, exponential linear regression and PLSR allowed correct age evaluation for five fingermarks out of eight.

The age of the eight test fingermarks was also estimated using LR calculations based on a Bayesian network in order to evaluate one pair of alternative temporal hypotheses ( $\leq 10$ or $\geq 14$ days old). The obtained LRs showed promising results when the storage conditions were known. Significant results were mainly obtained with fingermarks exposed to light. The proposed probabilistic approach should thus be further improved and tested on more data. Combining additional and alternative ageing parameters and influence factors may also yield better results.

It is particularly important to highlight that the variability of fingermark residue would probably never be completely erased by data processing and thus, fingermark dating development has to consider ways to avoid and reduce it. This study revealed difficulties to reduce the variability due to donor, substrate and enhancement techniques using the selected lipid aging parameters and models. The value of these known factors should thus be included in the aging models built for dating purposes for each new case. The deposition moment and pressure as well as the temperature and lighting conditions also brought variability to fingermark residues, but relevant aging models could still be built.

To conclude, this study showed that reproducible aging modelling and correct fingermark dating could be obtained based on fingermark residue exposed to unknown influence factors. However, the aging variability of the targeted lipids was also clearly shown. These results should thus be completed by other studies aiming at collecting more data in order to further test the robustness of the proposed models. Other target compounds, analytical techniques or processing steps could also be envisaged, opening a very large investigation field. The produced models should finally be properly optimised and validated before any application in real caseworks could be envisaged.

\section{References}

[1] J. Berry, D.A. Stoney, Chapter 1: the history and development of fingerprinting, in: H.C. Lee, R.E. Gaensslen, B. Raton (Eds.),Advances in Fingerprint Technology, CRC Press, 2001

[2] S. Cole, Suspect Identities: A History of Fingerprinting and Criminal Identification, Harvard University Press, 2001.

[3] C. Sengoopta, Imprint of the Raj - How Fingerprinting was Born in Colonial India, Macmillan, London, 2003.

[4] C. Champod, C. Lennard, P. Margot, M. Stoilovic, Fingerprints and Other Ridge Skin Impressions, 1st ed.: CRC Press, 2004.

[5] P. Margot, A question of time, Sci. Justice 40 (2) (2000) 64-71.

[6] C. Weyermann, O. Ribaux, Situating forensic traces in time, Sci. Justice 52 (2) (2012) 68-75.

[7] A. Girod, C. Weyermann, La datation des traces digitales (partie I): revue critique, Revue internationale de criminologie et de police technique 66 (3) (2013) 364-377.

[8] A. Girod, C. Roux, C. Weyermann, La datation des traces digitales (partie II): proposition d'une approche formelle, Revue internationale de criminologie et de police technique 68 (2) (2014) 226-249.

[9] A. Girod, R. Ramotowski, S. Lambrechts, P. Misrielal, M. Aalders, C. Weyermann, Fingermark dating: legal considerations, review of the literature and practical propositions, Forensic Sci. Int. (2015) (submitted for publication).

[10] R.D. Olsen, Chemical dating techniques for latent fingerprints: a preliminary report, The Identification News, 10-12, 1987.

[11] K. Wertheim, Fingerprint age determination: is there any hope? J Forensic Identif. 53 (1) (2003) 42-49.

[12] A. Girod, R. Ramotowski, C. Weyermann, Composition of fingermark residue: a qualitative and quantitative review, Forensic Sci. Int. 223 (1-3) (2012) 10-24.

[13] R.S. Ramotowski, Composition of latent print residues, in: H.C. Lee, R.E. Gaensslen, B. Raton, C.R.C. Press (Eds.),Advances in Fingerprint Technology 2001, pp. 63-104.

[14] S.J. Cadd, M. Islam, P. Manson, S. Bleay, Fingerprint composition and aging: a literature review, Sci. Justice 55 (4) (2015) 219-238.

[15] R.S. Ramotowski, Chapter 3: composition of latent print residues, in: H.C. Lee, R.E. Gaensslen, B. Raton (Eds.), Advances in Fingerprint Technology, CRC Press, 2001.

[16] N.E. Archer, Y. Charles, J.A. Elliott, S. Jickells, Changes in the lipid composition of latent fingerprint residue with time after deposition on a surface, Forensic Sci. Int. 154 (2-3) (2005) 224-239.

[17] C. Weyermann, C. Roux, C. Champod, initial results on the composition of fingerprints and its evolution as a function of time by GC/MS analysis, J. Forensic Sci. 56 (1) (2011) 102-108

[18] G.M. Mong, C.E. Petersen, T.R.W. Clauss, Advanced Fingerprint Analysis Project Final Report - Fingerprint Constituents, Pacific Northwest National Laboratory, 1999.

[19] A. Koenig, A. Girod, C. Weyermann, Identification of wax esters in fingermark residues by GC/MS and their potential use as aging parameters, J. Forensic. Identif. 61 (6) (2011) 652-676.

[20] A. Girod, C. Weyermann, Lipid composition of fingermark residue and donor classification using GC/MS, Forensic Sci. Int. 238 (2014) 68-82.

[21] C. Weyermann, J. Almog, J. Bügler, A.A. Cantu, Minimum requirements for application of ink dating methods based on solvent analysis in casework, Forensic Sci. Int. $210(1-3)$ (2011) 52-62.

[22] C. Weyermann, B. Schiffer, P. Margot, A logical framework to ballpoint ink dating interpretation, Sci. Justice 48 (3) (2008) 118-125.

[23] M. Gallidabino, C. Weyermann, F.S. Romolo, F. Taroni, Estimating the time since discharge of spent cartridges: a logical approach for interpreting the evidence, Sci. Justice 53 (1) (2013) 41-48.

[24] J.N. Miller, J.C. Miller, Statistics and chemometrics for analytical chemistry, Pearson, fifth ed.Prentice Hall, 2005.

[25] D.L. Massart, B.G.M. Vandeginste, L.M.C. Buydens, S. De Jong, P.J. Lewi, J. SmeyersVerbeke, Data Handling in Science and Technology 20A: Handbook of Chemometrics and Qualimetrics, Part A, Elsevier, Amsterdam, 1997. 
[26] B.G.M. Vandeginste, D.L. Massart, L.M.C. Buydens, S. De Jong, P.J. Lewi, J. SmeyersVerbeke, Data Handling in Science and Technology 20B: Handbook of Chemometrics and Qualimetrics, Part B, Elsevier, Amsterdam, 1997.

[27] S. Lociciro, P. Esseiva, P. Hayoz, L. Dujourdy, F. Besacier, P. Margot, Cocaine profiling for strategic intelligence, a cross-border project between France and Switzerland, part II: validation of the statistical methodology for the profiling of cocaine, Forensic Sci. Int. 177 (2-3) (2008) 199-206.

[28] R. Marquis, C. Weyermann, C. Delaporte, P. Esseiva, L. Aalberg, F. Besacier, et al., Drug intelligence based on MDMA tablets data: 2. Physical characteristics profiling, Forensic Sci. Int. 178 (1) (2008) 34-39.

[29] C. Weyermann, R. Marquis, C. Delaporte, P. Esseiva, E. Lock, L. Aalberg, et al., Drug intelligence based on MDMA tablets data: I. Organic impurities profiling, Forensic Sci. Int. 177 (1) (2008) 11-16.

[30] C. Weyermann, L. Bucher, P. Majcherczyk, A statistical methodology for the comparison of blue gel pen inks analyzed by laser desorption/ionization mass spectrometry, Sci. Justice 51 (3) (2011) 122-130.

[31] C. Weyermann, L. Bucher, P. Majcherczyk, W. Mazzella, C. Roux, P. Esseiva, Statistical discrimination of black gel pen inks analysed by laser desorption/ionization mass spectrometry, Forensic Sci. Int. 217 (1-3) (2012) 127-133.

[32] O. Guéniat, P. Esseiva, Le profilage de l'héroïne et de la cocaïne: Une méthodologie moderne de lutte contre le trafic illicite: PPUR, 2005 Lausanne.

[33] J. Broséus, F. Anglada, P. Esseiva, The differentiation of fibre- and drug type cannabis seedlings by gas chromatography/mass spectrometry and chemometric tools, Forensic Sci. Int. 200 (1-3) (2010) 87-92.
[34] J. Broséus, M. Vallat, P. Esseiva, Multi-class differentiation of cannabis seedlings in a forensic context, Chemom. Intell. Lab. Syst. 107 (2) (2011) 343-350.

[35] I.T. Jolliffe, Principal Component Analysis, second ed. Springer Series in Statistics, 2002.

[36] K.H. Esbensen, D. Guyot, F. Westad, L.P. Houmøller, Multivariate Data Analysis in Practice: An Introduction to Multivariate Data Analysis and Experimental Design, fifth ed. Camo Process AS, Oslo, 2002.

[37] C.G.G. Aitken, F. Taroni, Statistics and the Evaluation of Evidence for Forensic Scientists, second ed. John Wiley \& Sons, Chichester, England, 2004.

[38] F. Taroni, S. Bozza, A. Biedermann, P. Garbolino, C.G.G. Aitken, Data Analysis in Forensic Science: A Bayesian Decision Perspective, John Wiley \& Sons, Chichester, England, 2010.

[39] F. Taroni, A. Biederman, S. Bozza, P. Garbolino, C.G.G. Aitken, Bayesian Networks for Probabilistic Inference and Decision Analysis in Forensic Science, second ed. John Wiley \& Sons, Chichester, 2014.

[40] F. Taroni, C. Aitken, P. Garbolino, A. Biedermann, ). Bayesian Networks and Probability Inference in Forensic Science, John Wiley \& Sons, Ltd, Chichester, 2006 B.

[41] E. Sironi, M. Gallidabino, C. Weyermann, F. Taroni, Probabilistic graphical models to deal with age estimation of living persons, Int. J. Legal Med. (2015).

[42] A. Biedermann, F. Taroni, Bayesian networks and probabilistic reasoning about scientific evidence when there is a lack of data, Forensic Sci. Int. 157 (2-3) (2006) 163-167. 